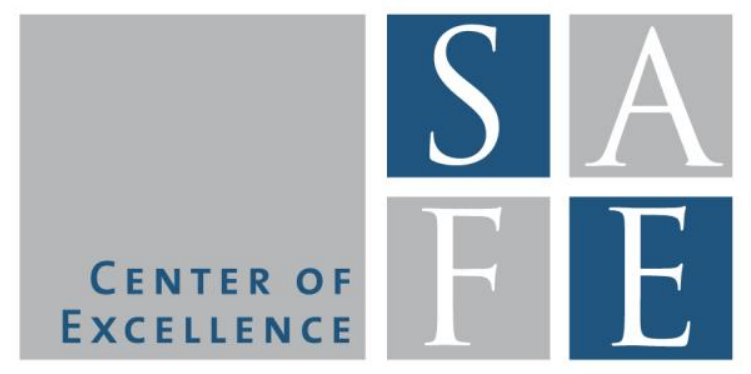

WORKING PAPER SERIES

Holger Kraft - Frank Thomas Seifried

\title{
Stochastic Differential Utility as the Continuous-Time Limit of Recursive Utility
}

SAFE Working Paper Series No. 17

Center of Excellence SAFE Sustainable Architecture for Finance in Europe A cooperation of the Center for Financial Studies and Goethe University Frankfurt 


\title{
STOCHASTIC DIFFERENTIAL UTILITY AS THE CONTINUOUS-TIME LIMIT OF RECURSIVE UTILITY
}

\author{
HOLGER KRAFT \\ Holger Kraft, Department of Finance, Goethe University \\ Grueneburgplatz 1, 60323 Frankfurt am Main, Germany
}

FRANK THOMAS SEIFRIED

Frank Seifried, Department of Mathematics, University of Kaiserslautern 67653 Kaiserslautern, Germany

\begin{abstract}
We establish a convergence theorem that shows that discrete-time recursive utility, as developed by Kreps and Porteus (1978), converges to stochastic differential utility, as introduced by Duffie and Epstein (1992), in the continuous-time limit of vanishing grid size.
\end{abstract}

KEY WORDS: Stochastic differential utility, recursive utility, convergence, backward stochastic differential equation.

JEL Classification: D81, D91

Date: May 10, 2013 (this version); June 28, 2011 (first version).

We thank Nicole Bäuerle, Dirk Becherer, Michael Brennan, Simone Cerreia-Vioglio, Darrell Duffie, Christian Hellwig, Ulrich Horst, Günter Last, Dilip Madan, Fabio Maccheroni, Massimo Marinacci, Claus Munk, Mark Schroder and Costis Skiadas for helpful comments and suggestions. We also thank participants of the De Finetti Risk Seminar at Bocconi, the Bachelier Finance Society World Congress in Sydney, the $10^{\text {th }}$ Probability and Stochastics Days in Mainz, and the seminars of HU and TU Berlin and Karlsruhe Institute of Technology for helpful discussions. All remaining errors are of course our own. Holger Kraft gratefully acknowledges financial support by Deutsche Forschungsgemeinschaft (DFG). 


\section{INTRODUCTION}

Recursive preferences have become important in a large number of applications including macroeconomic and finance theory, and, in particular, equilibrium asset pricing. In the literature, the notion of stochastic differential utility has been accepted as the continuous-time analog of recursive utility. This correspondence implicitly underlies a large number of applications of stochastic differential utility. However, a rigorous proof of this connection has not been given yet. This paper closes that gap: We show that, in a general semimartingale framework and under standard assumptions on the aggregator, stochastic differential utility is the continuous-time limit of recursive utility.

It is well-known that the standard discounted expected utility paradigm restricts the relationship between preferences for smoothing across time and across states. To address this issue, the concept of discrete-time recursive utility was developed by Kreps and Porteus (1978), Epstein and Zin (1989), Weil (1990), and others, making it possible to disentangle risk aversion from the elasticity of intertemporal substitution. Stochastic differential utility was introduced by Epstein (1987) in a deterministic setting and by Duffie and Epstein (1992) in a stochastic setting as a continuous-time version of recursive utility. Epstein (1987), Duffie and Epstein (1992) and the subsequent literature define stochastic differential utility axiomatically in continuous time, but do not establish a rigorous connection to discrete-time recursive utility. Heuristic links to recursive utility are provided in Duffie and Epstein (1992), Svensson (1989) and Skiadas (2008).

The limitations of discounted expected utility are particularly apparent in an important area of research: the theory of equilibrium asset pricing. The implications of expected utility are known to be incompatible with various stylized facts in empirical findings; for instance, the excess return of stocks implied by expected utility is much too high for realistic risk aversion parameters (this is the "equity premium puzzle" of Mehra and Prescott (1985)). In the last 25 years, recursive preferences have therefore become a key ingredient in the asset pricing literature; see, e.g., Duffie and Epstein (1992b), Obstfeld (1994), Tallarini (2000), Bansal and Yaron (2004), Uhlig (2007), Hansen, Heaton, and Li (2008), Guvenen (2009), Kaltenbrunner and Lochstoer (2010), Gabaix (2012), Borovička, Hansen, Hendricks, and Scheinkman (2011) and Wachter (2011). These papers demonstrate that recursive preferences are a highly relevant modeling tool which allows researchers to address various open questions in economics and finance. Despite their empirical relevance, the literature lacks a rigorous result relating discrete-time recursive utility to stochastic differential utility. Thus, so far discrete-time and continuous-time models coexist (e.g., Bansal and Yaron (2004) is a recent discrete-time model, while Wachter (2011) is set in continuous time). ${ }^{1}$

Our main result (Theorem 4.1) shows that, under general conditions, the KrepsPorteus recursive utilities associated to a sequence of approximating consumption plans converge to the stochastic differential utility of the limiting consumption plan as the

\footnotetext{
${ }^{1}$ This is in stark contrast to, for instance, the theory of option pricing: There are several results, including, e.g., Cox, Ross, and Rubinstein (1979) and Duffie and Protter (1992), on the convergence of option prices in discrete-time models to their continuous-time counterparts.
} 
grid size tends to zero. We also provide a rigorous justification of a classical formula, first derived by Epstein (1987) in a deterministic setting, that is commonly used to obtain a continuous-time aggregator from its discrete-time counterpart. Theorem 4.1 is based on assumptions that may be violated in some specific applications. We therefore also provide a convergence theorem that applies to bounded consumptions plans under milder assumptions on the aggregator. In particular, we apply our convergence results to the empirically important Epstein-Zin-Weil parametrization of recursive utility. Finally, we provide a general sufficient condition for recursive utility with a nonstandard certainty equivalent to converge to stochastic differential utility.

The remainder of this paper is structured as follows: In Section 2 we fix the framework for the paper's analysis. Section 3 briefly reviews recursive and stochastic differential utility. In Section 4 we present our main convergence result. Section 5 contains the proof. Section 6 establishes a more general convergence result for bounded consumption plans. In Section 7 we provide a basis for further research on convergence with nonstandard certainty equivalents, and Section 8 concludes.

\section{Mathematical Setting}

We work on a filtered probability space $(\Omega, \mathfrak{A}, \mathfrak{F}, \mathbb{P})$ whose filtration $\mathfrak{F}=\left\{\mathfrak{F}_{t}\right\}_{t \in[0, T]}$ satisfies the "usual conditions" of right-continuity and $\mathbb{P}$-completeness. We assume that $\mathfrak{F}_{0}$ is $\mathbb{P}$-trivial, and whenever $M=\left\{M_{t}\right\}_{t \in[0, T]}$ is an $(\mathfrak{F}, \mathbb{P})$-martingale, we work with a càdlàg version of $M$.

Consumption Plans. Let $C \subseteq \mathbb{R}$ be an interval modeling the consumption space (typically, $C \subseteq[0, \infty)$ ). We call a consumption rate process $c=\left\{c_{t}\right\}_{t \in[0, T]}$ feasible if $c$ is $\mathrm{C}$-valued and $\mathfrak{F}$-progressively measurable with

$$
\mathbb{E}\left[\sup _{t \in[0, T]}\left|c_{t}\right|^{2}\right]<\infty .
$$

We denote by $\mathcal{A}$ the class of feasible consumption plans. Moreover we fix a terminal payoff $X$, given as a $C$-valued $\mathfrak{F}_{T}$-measurable random variable with $\mathbb{E}\left[|X|^{2}\right]<\infty$.

Approximating Sequences. We study an arbitrary but fixed sequence $\left\{\pi^{N}\right\}_{N \in \mathbb{N}}$ of partitions $\pi^{N}=\left[t_{0}^{N}, \ldots, t_{N}^{N}\right]$ of $[0, T]$ where $^{2} 0=t_{0}^{N} \leq t_{1}^{N} \leq \cdots \leq t_{N}^{N}=T$ and $\pi^{N} \subseteq \pi^{N+1}$. We set $\Delta_{k}^{N} \triangleq t_{k+1}^{N}-t_{k}^{N}, k=0, \ldots, N-1$ and assume that

$$
\Delta^{N} \triangleq \max _{k=0, \ldots, N-1} \Delta_{k}^{N} \rightarrow 0 \text { as } N \rightarrow \infty \text {. }
$$

A sequence $\left\{c^{N}\right\}_{N \in \mathbb{N}}$ of $\mathrm{C}$-valued adapted processes is said to be an approximating sequence for the feasible plan $c$ if $c^{N}$ is right-continuous and piecewise constant on $\pi^{N}$ for each $N \in \mathbb{N}$ and ${ }^{3}$

$$
c^{N} \rightarrow c \text { a.e. on }[0, T] \times \Omega \text { as } N \rightarrow \infty
$$

subject to the integrability condition

$$
\mathbb{E}\left[\sup _{N \in \mathbb{N}, t \in[0, T]}\left|c_{t}^{N}\right|^{2}\right]<\infty .
$$

Note that (2) and (3) imply (1) for a suitable modification of $c$.

\footnotetext{
${ }^{2}$ To simplify notation, we assume that $\pi^{N}$ has exactly $N+1$ grid points.

${ }^{3}[0, T] \times \Omega$ is endowed with the product measure $\mathrm{d} t \otimes \mathbb{P}$.
} 
Examples of Approximating Sequences. (a) If $c \in \mathcal{A}$ has paths with left and right limits and $c^{N}$ is the discretization of $c$ along $\pi^{N}$, i.e.

$$
c_{t}^{N} \triangleq c_{t_{k}^{N}} \text { for } t \in\left[t_{k}^{N}, t_{k+1}^{N}\right), k=0, \ldots, N-1
$$

then $\left\{c^{N}\right\}_{N \in \mathbb{N}}$ constitutes an approximating sequence.

(b) The approach in (a) applies in particular to piecewise-constant consumption plans. For instance, $c$ may be represented by a continuous-time Markov chain. In this case, $c^{N}$ represents the canonical tree-type approximation of $c$.

(c) If $\left\{c^{N}\right\}_{N \in \mathbb{N}}$ is an approximating sequence for $c$, then $\left\{\bar{c}^{N}\right\}_{N \in \mathbb{N}}$ is also one where $\bar{c}^{N}$ is the space discretization of $c^{N}$ along $\zeta^{N}$, i.e.

$$
\bar{c}_{t}^{N} \triangleq z_{k}^{N} \text { if } c_{t}^{N} \in\left[z_{k}^{N}, z_{k+1}^{N}\right) \text { for } t \in[0, T]
$$

with a sequence $\left\{\zeta^{N}\right\}_{N \in \mathbb{N}}$ of partitions $\zeta^{N}=\left[z_{0}^{N}, \ldots, z_{N}^{N}\right]$ of the consumption space C such that $\max _{k=0, \ldots, N-1}\left|z_{k+1}^{N}-z_{k}^{N}\right| \rightarrow 0$ as $N \rightarrow \infty$.

(d) In combination with (a), case (c) covers binomial tree-type approximations to Brownian models, given suitable integrability conditions.

Note that (b) and (d) correspond to the settings analyzed by Skiadas (2013).

\section{Recursive Utility and Stochastic Differential Utility}

To model preferences for intertemporal consumption in a stochastic setting, we are generally interested in mappings of the form

$$
\mathfrak{v}: \mathcal{A} \rightarrow \mathbb{R}, \quad c \mapsto \mathfrak{v}(c)
$$

such that $c$ is preferred to $c^{\prime}$ if and only if $\mathfrak{v}(c)>\mathfrak{v}\left(c^{\prime}\right)$. If such a representation is available, the functional $\mathfrak{v}$ is referred to as a utility index. Clearly, if $\mathfrak{v}$ and $\tilde{\mathfrak{v}}$ are utility indices and there exists a strictly increasing function $\varphi$ such that $\tilde{\mathfrak{v}}=\varphi \circ \mathfrak{v}$, then they describe the same preferences; in this case, $\mathfrak{v}$ and $\tilde{\mathfrak{v}}$ are said to be ordinally equivalent.

\subsection{ReCURsive UtiLity}

Recursive utility is a paradigm to construct utility indices in a discrete-time framework. Following Kreps and Porteus (1978), its two main components are

(i) a discrete-time intertemporal aggregator $W$, i.e. a mapping

$$
W:[0, T] \times \mathrm{C} \times \mathrm{C} \rightarrow \mathrm{C}, \quad(\Delta, c, v) \mapsto W(\Delta, c, v)
$$

with $W(0, c, v)=v$ for all $(c, v) \in \mathrm{C} \times \mathrm{C}$; and

(ii) a certainty equivalent $\mathfrak{m}$, i.e. a mapping

$$
\mathfrak{m}: \mathcal{M}(\mathrm{C}) \rightarrow \mathrm{C}, \quad \mu \mapsto \mathfrak{m}(\mu)
$$

such that $\mathfrak{m}\left(\delta_{c}\right)=c$ for all $c \in \mathrm{C}$, where $\mathcal{M}(\mathrm{C})$ denotes the space of all probability distributions on $\mathrm{C}$ such that $\int_{\mathrm{C}}|v|^{2} \mu(\mathrm{d} v)<\infty$ and $\delta_{c}$ is the Dirac measure at $c$. 
The pair $(W, \mathfrak{m})$ is referred to as a (discrete-time) aggregator. The current utility of consumption $c \Delta$ during a time interval of length $\Delta$ and a random payoff with distribution $\mu$ at the end of this interval is computed via the aggregation

$$
(\Delta, c, \mu) \mapsto W(\Delta, c, \mathfrak{m}(\mu)) .
$$

More precisely, given an aggregator $(W, \mathfrak{m})$ the recursive utility index of a feasible consumption stream $c^{N}$ that is piecewise constant on $\pi^{N}$ is given by

$$
\mathfrak{v}^{N}\left(c^{N}\right) \triangleq V_{0}^{N}
$$

with the continuation value process $V^{N}=\left\{V_{t_{k}^{N}}^{N}\right\}_{k=0, \ldots, N-1}$ defined recursively via ${ }^{4}$

$$
V_{t_{k}^{N}}^{N}=W\left(\Delta_{k}^{N}, c_{t_{k}^{N}}^{N}, \mathfrak{m}\left(\mathcal{L}_{t_{k}^{N}}\left(V_{t_{k+1}^{N}}^{N}\right)\right)\right), k=N-1, \ldots, 0, \quad V_{t_{N}^{N}}^{N}=X .
$$

Here $\mathcal{L}_{t}(Y)$ denotes the conditional distribution, given time- $t$ information $\mathfrak{F}_{t}$, of the random variable $Y$. Thus at each time $t_{k}^{N}$ the agent aggregates current consumption $c_{t_{k}^{N}}^{N}$ with the time- $t_{k}^{N}$ certainty equivalent $\mathfrak{m}\left(\mathcal{L}_{t_{k}^{N}}\left(V_{t_{k+1}^{N}}^{N}\right)\right)$ of consumption starting in the next period.

The recursion (4) shows that $W$ models intertemporal preferences, while $\mathfrak{m}$ captures risk preferences. In Sections $3-6$ we assume that $\mathfrak{m}$ can be represented in the form characterized by Kreps and Porteus (1978):

$$
\mathfrak{m}(\mu)=u^{-1}\left(\int_{\mathrm{C}} u(v) \mu(\mathrm{d} v)\right), \mu \in \mathcal{M}(\mathrm{C})
$$

with $u: \quad C \rightarrow \mathbb{R}$ a strictly increasing continuous function of linear growth. ${ }^{5}$ The interpretation is that preferences between (atemporal) random payoffs $Y$ and $Z$ are characterized by their expected utilities $\mathbb{E}[u(Y)]$ and $\mathbb{E}[u(Z)]$.

Normalization. The transformation $v \mapsto \tilde{v} \triangleq u(v)$ leads to the aggregation

$$
(\Delta, c, \mu) \mapsto \tilde{W}\left(\Delta, c, \mathfrak{m}_{0}(\mu)\right)
$$

corresponding to the aggregator $\left(\tilde{W}, \mathfrak{m}_{0}\right)$ where

$$
\tilde{W}:[0, T] \times \mathrm{C} \times \mathrm{V} \rightarrow \mathrm{V}, \quad \tilde{W}(\Delta, c, v) \triangleq u\left(W\left(\Delta, c, u^{-1}(v)\right)\right)
$$

and $\mathfrak{m}_{0}$ denotes the risk-neutral certainty equivalent on $\mathrm{V} \triangleq u(\mathrm{C})$,

$$
\mathfrak{m}_{0}(\mu) \triangleq \int_{\mathrm{V}} v \mu(\mathrm{d} v), \mu \in \mathcal{M}(\mathrm{V}) .
$$

The backward recursion (4) for $\tilde{V}^{N}=\left\{\tilde{V}_{t_{k}^{N}}^{N}\right\}_{k=0, \ldots, N}, \tilde{V}_{t_{k}^{N}}^{N}=u\left(V_{t_{k}^{N}}^{N}\right)$ now reads

$$
\tilde{V}_{t_{k}^{N}}^{N}=\tilde{W}\left(\Delta_{k}^{N}, c_{t_{k}^{N}}^{N}, \mathbb{E}_{t_{k}^{N}}\left[\tilde{V}_{t_{k+1}^{N}}^{N}\right]\right), k=N-1, \ldots, 0, \quad \tilde{V}_{t_{N}^{N}}^{N}=\xi
$$

and we set $\tilde{\mathfrak{v}}^{N}\left(c^{N}, X\right) \triangleq \tilde{V}_{0}^{N}$. Here $\mathbb{E}_{t}$ denotes conditional expectation given $\mathfrak{F}_{t}$ and $\xi \triangleq u(X)$. Importantly, the aggregator $(W, \mathfrak{m})$ describes (via $(4))$ the same preferences as $\left(\tilde{W}, \mathfrak{m}_{0}\right)$ (via $\left.(6)\right)$ : The corresponding utility indices are linked via $\tilde{\mathfrak{v}}^{N}\left(c^{N}\right)=\tilde{V}_{0}^{N}=$ $u\left(V_{0}^{N}\right)=u\left(\mathfrak{v}^{N}\left(c^{N}\right)\right)$ so they are ordinally equivalent.

\footnotetext{
${ }^{4}$ Existence of recursive utility is guaranteed in our setting by conditions (A1), (A2), (A3) and (A4) below. We refer to Marinacci and Montrucchio (2010) for existence and uniqueness results under more general conditions.

${ }^{5}$ The growth condition ensures that $\mathfrak{m}$ is well-defined. If this is guaranteed by other means, the condition can be relaxed; see also Section 6 .
} 
Remark. The terminal payoff $X$ is transformed to the terminal utility $\xi=u(X)$. Since $u$ is of linear growth, we also have $\mathbb{E}\left[|\xi|^{2}\right]<\infty$.

The above normalization procedure is feasible for every certainty equivalent of KrepsPorteus type (5). Therefore, in the following we directly work with a normalized aggregator $\left(W, \mathfrak{m}_{0}\right)$ (or simply $W$, for short). Thus we study the discrete-time recursive utility index given by

$$
\mathfrak{v}^{N}\left(c^{N}\right) \triangleq V_{0}^{N} \text { for } c^{N} \in \mathcal{A} \text { piecewise constant on } \pi^{N}
$$

where $V^{N}=\left\{V_{t_{k}^{N}}^{N}\right\}_{k=0, \ldots, N}$ satisfies the backward aggregation

$\left(\operatorname{Rec}^{N}\right) \quad V_{t_{k}^{N}}^{N}=W\left(\Delta_{k}^{N}, c_{t_{k}^{N}}^{N}, \mathbb{E}_{t_{k}^{N}}\left[V_{t_{k+1}^{N}}^{N}\right]\right), k=0, \ldots, N-1, \quad V_{t_{N}^{N}}^{N}=\xi$.

Here the terminal utility $\xi$, represented by an $\mathfrak{F}_{T}$-measurable random variable with $\mathbb{E}\left[|\xi|^{2}\right]<\infty$, and the temporal aggregator $W:[0, T] \times \mathbf{C} \times \mathbb{R} \rightarrow \mathbb{R}$ are given. ${ }^{6}$

Remark. If $W$ is additive in the sense that $W(\Delta, c, v)=\alpha \Delta u(c)+(1-\delta \Delta) v$, we recover the classical special case of discounted expected utility:

$$
\mathfrak{v}^{N}\left(c^{N}\right)=V_{0}^{N}=\mathbb{E}\left[\alpha \sum_{k=0}^{N-1} \beta_{k}^{N} u\left(c_{t_{k}^{N}}^{N}\right) \Delta_{k}^{N}+\beta_{N}^{N} u(X)\right]
$$

where $\xi=u(X)$ and $\beta_{k}^{N} \triangleq \prod_{j=0}^{k-1}\left(1-\delta \Delta_{j}^{N}\right)$ is the discrete time- $t_{k}^{N}$ discount factor.

\subsection{Stochastic Differential Utility}

Stochastic differential utility was introduced by Duffie and Epstein (1992) as a continuoustime analog of recursive utility. Although they provide a heuristic connection to recursive utility, Duffie and Epstein (1992) give a rigorous definition of stochastic differential utility in continuous time only: For a feasible consumption plan $c$ and a given (continuous-time) aggregator $f: \mathbf{C} \times \mathbb{R} \rightarrow \mathbb{R}$ they define

$$
\mathfrak{v}(c) \triangleq V_{0}
$$

where $\xi$ is an $\mathfrak{F}_{T}$-measurable random variable and the process $V=\left\{V_{t}\right\}_{t \in[0, T]}$ is given by the backward stochastic differential equation (BSDE)

$$
V_{t}=\mathbb{E}_{t}\left[\int_{t}^{T} f\left(c_{s}, V_{s}\right) \mathrm{d} s+\xi\right], t \in[0, T]
$$

or alternatively, in equivalent differential notation,

$$
\mathrm{d} V_{t}=-f\left(c_{t}, V_{t}\right) \mathrm{d} t+\mathrm{d} M_{t}, \quad V_{T}=\xi
$$

with $M=\left\{M_{t}\right\}_{t \in[0, T]}$ an $(\mathfrak{F}, \mathbb{P})$-martingale. ${ }^{7}$

Remark. Similarly as in the discrete-time case, if $f$ takes the additive form $f(c, v)=$ $\alpha u(c)-\delta v$ and $\xi=u(X)$ we obtain standard expected utility with

$$
V_{t}=\mathbb{E}_{t}\left[\alpha \int_{t}^{T} \mathrm{e}^{-\delta s} u\left(c_{s}\right) \mathrm{d} s+\mathrm{e}^{-\delta T} u(X)\right], t \in[0, T] .
$$

\footnotetext{
${ }^{6}$ We assume that $W(\Delta, c, \cdot)$ is defined on $\mathbb{R}$. This is a technical requirement that can be relaxed, see Section 6.

${ }^{7}$ See Proposition A.2 in Appendix A for conditions on $f$ that ensure existence and uniqueness.
} 


\section{The Convergence Result}

Throughout Sections 4 and 5 we suppose that a normalized discrete-time aggregator $W$ and a terminal utility $\xi$ with $\mathbb{E}\left[|\xi|^{2}\right]<\infty$ are given. For a feasible consumption plan $c$ and an approximating sequence $\left\{c^{N}\right\}_{N \in \mathbb{N}}$ we study the sequence $\left\{V^{N}\right\}_{N \in \mathbb{N}}$ of recursive utility processes constructed via $\left(\operatorname{Rec}^{N}\right)$ and the process $V$ given by (SDU). When there is a risk of confusion, we denote $V^{N}$ by $V^{N}\left(c^{N}\right)$ and $V$ by $V(c)$ to highlight the dependencies on $c^{N}$ and $c$, respectively.

Conditions (A1), (A2), (A3), (A4). We impose the following regularity conditions on the aggregator $W$ : There is a function $f: C \times \mathbb{R} \rightarrow \mathbb{R}$ such that

$$
\begin{aligned}
& W(\Delta, c, v)=v+f(c, v) \Delta+\varepsilon(\Delta, c, v) \Delta \\
& \quad \text { with }|\varepsilon(\Delta, c, v)| \leq h(\Delta)(1+|c|+|v|),(\Delta, c, v) \in[0, T] \times C \times \mathbb{R} \\
& |f(c, v)-f(c, w)| \leq L|v-w| \text { for } c \in \mathrm{C}, v, w \in \mathbb{R} \\
& |f(c, 0)| \leq K(1+|c|) \text { for } c \in \mathrm{C} \\
& f(\cdot, v) \text { is continuous on } \mathrm{C} \text { for } v \in \mathbb{R}
\end{aligned}
$$

where $K, L>0$ are constants and $h:[0, T] \rightarrow[0, \infty)$ is a continuous increasing function with $h(0)=0$. In Theorem 4.1 below we identify $f$ as the continuoustime aggregator corresponding to $W$. Condition (A1) is a regularity condition for the discrete-time aggregator $W$; it essentially requires that $W$ grows linearly in $\Delta$ at $\Delta=0$. Note that (A1) is always satisfied for additive utility. (A1) implies in particular that $W(0, c, v)=v$ and that $f$ can be derived from $W$ via

$$
\frac{\partial W}{\partial \Delta}(0, c, v)=f(c, v) \text { for all }(c, v) \in \mathbf{C} \times \mathbb{R} .
$$

Conditions (A2) and (A3) are Lipschitz and linear growth conditions for the aggregator in (SDU) that are standard in the literature on BSDEs; see, e.g., Duffie and Epstein (1992) and El Karoui, Peng, and Quenez (1997). Condition (A4) is natural if a welldefined continuous-time limit is to be achieved.

Remark. Epstein (1987) and Duffie and Epstein (1992) specify the discrete-time aggregator as a smooth function $W:[0, T] \times \mathbf{C} \times \mathbb{R} \rightarrow \mathbb{R}$ such that $W(0, c, v)=v$ and define the continuous-time generator $\tilde{f}$ via $\tilde{f}(c, v) \triangleq \frac{\partial W}{\partial \Delta}(0, c, v) / \frac{\partial W}{\partial v}(0, c, v)$. In our setting this definition coincides with $(8)$ since $\frac{\partial W}{\partial v}(0, c, v)=1$.

Recursive Utility in Continuous Time. Let $V^{N}=\left\{V_{t_{k}^{N}}^{N}\right\}_{k=0, \ldots, N}$ denote the continuation value process associated to the consumption plan $c^{N} \in \mathcal{A}$ on the time grid $\pi^{N}$ via $\left(\operatorname{Rec}^{N}\right)$. For the analysis of convergence we extend the definition of $V^{N}$ to the whole time interval $[0, T]$ such that $V_{t}^{N}$ remains unaltered for $t \in \pi^{N}$ :

$$
V_{t}^{N} \triangleq W\left(\Delta_{k}^{N}, c_{t_{k}^{N}}^{N}, \mathbb{E}_{t}\left[V_{t_{k+1}^{N}}^{N}\right]\right) \text { for } t \in\left[t_{k}^{N}, t_{k+1}^{N}\right) \text { and } k=0, \ldots, N-1
$$

We now state the main result of this paper: Recursive utility converges to stochastic differential utility in the continuous-time limit. 
Theorem 4.1 (Convergence). Suppose that (A1), (A2), (A3) and (A4) hold and consider a feasible consumption plan $c \in \mathcal{A}$. Let $\left\{c^{N}\right\}_{N \in \mathbb{N}}$ be an approximating sequence and let the recursive utility process $V^{N}$ associated to $c^{N}$ be given by

$\left(\operatorname{Rec}^{N}\right) \quad V_{t}^{N} \triangleq W\left(\Delta_{k}^{N}, c_{t_{k}^{N}}^{N}, \mathbb{E}_{t}\left[V_{t_{k+1}^{N}}^{N}\right]\right), t \in\left[t_{k}^{N}, t_{k+1}^{N}\right), k=0, \ldots, N-1, \quad V_{T}^{N}=\xi$.

Moreover, let $V$ denote the stochastic differential utility process of c given by

$$
V_{t}=\mathbb{E}_{t}\left[\int_{t}^{T} f\left(c_{s}, V_{s}\right) \mathrm{d} s+\xi\right], t \in[0, T]
$$

where $W$ and $f$ are related via (8), i.e. $f(c, v)=\frac{\partial W}{\partial \Delta}(0, c, v)$. Then it follows that

$$
\left\|\sup _{t \in[0, T]}\left|V_{t}^{N}-V_{t}\right|\right\|_{2} \rightarrow 0 \text { as } N \rightarrow \infty \text {. }
$$

In particular, the associated utility indices satisfy

$$
\mathfrak{v}^{N}\left(c^{N}\right) \rightarrow \mathfrak{v}(c) \text { as } N \rightarrow \infty .
$$

\section{Proof of the Convergence Theorem}

We use the following extension of the definition of $f$ :

$$
f:[0, T] \times C \times \mathbb{R} \rightarrow \mathbb{R}, \quad f(\Delta, c, v) \triangleq \frac{W(\Delta, c, v)-v}{\Delta} .
$$

With this notation, (A1) can be restated as

$$
|f(\Delta, c, v)-f(c, v)| \leq h(\Delta)(1+|c|+|v|),(\Delta, c, v) \in[0, T] \times C \times \mathbb{R} .
$$

Conditions (A1), (A2) and (A3) imply that there is a constant $K_{1}>0$ with

$$
|f(\Delta, c, v)| \leq K_{1}(1+|c|+|v|) \text { for }(\Delta, c, v) \in[0, T] \times \mathbf{C} \times \mathbb{R} .
$$

Using (9) the recursion $\left(\operatorname{Rec}^{N}\right)$ can be equivalently reformulated as

$$
V_{t}^{N} \triangleq \mathbb{E}_{t}\left[V_{t_{k+1}^{N}}^{N}\right]+\Delta_{k}^{N} f\left(\Delta_{k}^{N}, c_{t_{k}^{N}}^{N}, \mathbb{E}_{t}\left[V_{t_{k+1}^{N}}^{N}\right]\right) \text { for } t \in\left[t_{k}^{N}, t_{k+1}^{N}\right)
$$

where $k=0, \ldots, N-1$ and $V_{t_{N}^{N}}^{N}=\xi$.

Outline of the Proof. Iterating (10) yields the representation ${ }^{8}$

$$
V_{t}^{N}=\mathbb{E}_{t}\left[\sum_{k=l}^{N-1} \Delta_{k}^{N} f\left(\Delta_{k}^{N}, c_{t_{k}^{N}}^{N}, \mathbb{E}_{t \vee t_{k}^{N}}\left[V_{t_{k+1}^{N}}^{N}\right]\right)+\xi\right], t \in\left[t_{l}^{N}, t_{l+1}^{N}\right) .
$$

Our goal is to show that $V^{N} \rightarrow V$ as $N \rightarrow \infty$, where

$$
V_{t}=\mathbb{E}_{t}\left[\int_{t}^{T} f\left(c_{s}, V_{s}\right) \mathrm{d} s+\xi\right] .
$$

Apparently $\left(\mathrm{SDU}^{N}\right)$ mimics a Riemann sum approximation of (SDU). This motivates the following strategy for the convergence proof: Starting from $\left(\mathrm{SDU}^{N}\right)$ with $f\left(\Delta_{k}^{N}, c_{t_{k}^{N}}^{N}, \mathbb{E}_{t \vee t_{k}^{N}}\left[V_{t_{k+1}^{N}}^{N}\right]\right)$, we replace

$$
f\left(\Delta_{k}^{N}, c_{t_{k}^{N}}^{N}, \mathbb{E}_{t \vee t_{k}^{N}}\left[V_{t_{k+1}^{N}}^{N}\right]\right) \stackrel{\text { Step } 1}{\rightarrow-\rightarrow} f\left(c_{t_{k}^{N}}^{N}, \mathbb{E}_{t \vee t_{k}^{N}}\left[V_{t_{k+1}^{N}}^{N}\right]\right) \stackrel{\text { Step } 2}{\rightarrow-\rightarrow} f\left(c_{t_{k}^{N}}^{N}, V_{t \vee t_{k}^{N}}^{N}\right)
$$

and, starting from (SDU) with $f\left(c_{s}, V_{s}\right)$ we replace

$$
\int_{t_{l}^{N}}^{T} f\left(c_{t_{k}^{N}}^{N}, V_{t_{k}^{N}}\right) \stackrel{\text { Step } 4}{\leftarrow--} \int_{t}^{T} f\left(c_{t_{k}^{N}}^{N}, V_{t_{k}^{N}}\right) \stackrel{\text { Step } 3}{\leftarrow--} \int_{t}^{T} f\left(c_{s}, V_{s}\right) .
$$

\footnotetext{
${ }^{8} s \vee t \triangleq \max \{s, t\}$. Note that $t \vee t_{k}^{N}=t_{k}^{N}$ for every summand except possibly the first.
} 
In the second part of the proof, we "meet halfway" and show with a Gronwall-Bellman argument that $\left(\mathrm{SDU}^{N}\right)$ with $f\left(c_{t_{k}^{N}}^{N}, V_{t_{k}^{N}}^{N}\right)$, i.e.

$$
V_{t}^{N}=\mathbb{E}_{t}\left[\sum_{k=l}^{N-1} \Delta_{k}^{N} f\left(c_{t_{k}^{N}}^{N}, V_{t \vee t_{k}^{N}}^{N}\right)+\xi\right]+\text { error, } t \in\left[t_{l}^{N}, t_{l+1}^{N}\right)
$$

and (SDU) with $f\left(c_{t_{k}^{N}}^{N}, V_{t_{k}^{N}}\right)$, i.e.

$$
V_{t}=\mathbb{E}_{t}\left[\int_{t_{l}^{N}}^{T} \tilde{f}_{s}^{N} \mathrm{~d} s+\xi\right]+\text { error }, t \in\left[t_{l}^{N}, t_{l+1}^{N}\right)
$$

where $\tilde{f}_{s}^{N} \triangleq f\left(c_{t_{k}^{N}}^{N}, V_{t_{k}^{N}}\right)$ for $s \in\left[t_{k}^{N}, t_{k+1}^{N}\right)$, are close and the error terms are small. Step 1 is crucial since in this step we carry out the transition from the discrete-time aggregator $W$ to its continuous-time counterpart $f$ via the linearization (A1). In Step 3 we switch from the continuous-time plan $c$ to the approximating sequence $\left\{c^{N}\right\}_{N \in \mathbb{N}}$. Steps 2 and 4 are intermediate steps. Finally, the Gronwall-Bellman argument establishes the key link between the recursive utility processes $V^{N}$ and the stochastic differential utility process $V$.

Remark. Our approach is related to stability results for BSDEs (such as, e.g., Antonelli (1996) and Coquet, Mackevičius, and Mémin (1998)) and to the recent literature on numerical approximations of BSDEs (see, e.g., Bouchard and Touzi (2004), Zhang (2004) and Bouchard and Elie (2008), among many others). We refer to Bouchard, Elie, and Touzi (2009) for an overview of this strand of research. This literature focuses on settings with finite martingale multiplicity (e.g., Brownian or jump-diffusion models). By contrast, in this paper we allow for a general semimartingale framework, so existing results are not directly applicable to our convergence problem. In addition, even with finite martingale multiplicity, recursive utility imposes a nonstandard discretization scheme (see, e.g., (10)): It involves nested conditional expectations and possibly pathdependent stochastic aggregators that depend explicitly on the time discretization.

Proof of Theorem 4.1. For reasons of readability, the first part of the proof is subdivided into lemmas that correspond to Steps 1, 2, 3 and 4 above. Necessary a priori estimates are deferred to Appendix A. Throughout this section, we consider a fixed consumption plan $c \in \mathcal{A}$ and an approximating sequence $\left\{c^{N}\right\}_{N \in \mathbb{N}}$ and assume that (A1), (A2), (A3) and (A4) are satisfied.

Step 1. By $\left(\mathrm{SDU}^{N}\right)$ we have

$$
V_{t}^{N}=\mathbb{E}_{t}\left[\sum_{k=l}^{N-1} \Delta_{k}^{N} f\left(c_{t_{k}^{N}}^{N}, \mathbb{E}_{t \vee t_{k}^{N}}\left[V_{t_{k+1}^{N}}^{N}\right]\right)+\xi\right]+R_{t}^{1, N} \text { for } t \in\left[t_{l}^{N}, t_{l+1}^{N}\right)
$$

where $R^{1, N}=\left\{R_{t}^{1, N}\right\}_{t \in[0, T]}$ is given by

$$
R_{t}^{1, N} \triangleq \mathbb{E}_{t}\left[\sum_{k=l}^{N-1} \Delta_{k}^{N}\left\{f\left(\Delta_{k}^{N}, c_{t_{k}^{N}}^{N}, \mathbb{E}_{t \vee t_{k}^{N}}\left[V_{t_{k+1}^{N}}^{N}\right]\right)-f\left(c_{t_{k}^{N}}^{N}, \mathbb{E}_{t \vee t_{k}^{N}}\left[V_{t_{k+1}^{N}}^{N}\right]\right)\right\}\right]
$$

for $t \in\left[t_{l}^{N}, t_{l+1}^{N}\right)$. This error term can be estimated as follows:

Lemma 5.1. There is a constant $K_{3}>0$ such that

$$
\left\|\sup _{t \in[0, T]}\left|R_{t}^{1, N}\right|\right\|_{2} \leq K_{3} h\left(\Delta^{N}\right) \text { for all } N \geq N_{0}\left(c,\left\{c^{N}\right\}_{N \in \mathbb{N}}\right) .
$$


Proof. For $t \in\left[t_{l}^{N}, t_{l+1}^{N}\right)$ we have from (A1') and the conditional triangle inequality

$$
\begin{aligned}
\left|R_{t}^{1, N}\right| & \leq \mathbb{E}_{t}\left[\sum_{k=l}^{N-1} \Delta_{k}^{N}\left|f\left(\Delta_{k}^{N}, c_{t_{k}^{N}}^{N}, \mathbb{E}_{t \vee t_{k}^{N}}\left[V_{t_{k+1}^{N}}^{N}\right]\right)-f\left(c_{t_{k}^{N}}^{N}, \mathbb{E}_{t \vee t_{k}^{N}}\left[V_{t_{k+1}^{N}}^{N}\right]\right)\right|\right] \\
& \leq h\left(\Delta^{N}\right) \mathbb{E}_{t}\left[\sum_{k=l}^{N-1}\left(1+\left|c_{t_{k}^{N}}^{N}\right|+\left|\mathbb{E}_{t \vee t_{k}^{N}}\left[V_{t_{k+1}^{N}}^{N}\right]\right|\right) \Delta_{k}^{N}\right] \\
& \leq h\left(\Delta^{N}\right) \mathbb{E}_{t}\left[\sum_{k=0}^{N-1}\left(1+\left|c_{t_{k}^{N}}^{N}\right|+\left|V_{t_{k+1}^{N}}^{N}\right|\right) \Delta_{k}^{N}\right] .
\end{aligned}
$$

Applying Doob's $L^{2}$-inequality to the $(\mathfrak{F}, \mathbb{P})$-martingale $M^{N}=\left\{M_{t}^{N}\right\}_{t \in[0, T]}$ defined by $M_{t}^{N} \triangleq \mathbb{E}_{t}\left[\sum_{k=0}^{N-1}\left(1+\left|c_{t_{k}^{N}}^{N}\right|+\left|V_{t_{k+1}^{N}}^{N}\right|\right) \Delta_{k}^{N}\right]$ for $t \in[0, T]$ it follows that

$$
\left\|\sup _{t \in[0, T]}\left|R_{t}^{1, N}\right|\right\|_{2} \leq h\left(\Delta^{N}\right)\left\|\sup _{t \in[0, T]}\left|M_{t}^{N}\right|\right\|_{2} \leq 2 h\left(\Delta^{N}\right)\left\|M_{T}^{N}\right\|_{2} .
$$

On the other hand, by Proposition A.1 and (27) in Appendix A we have

$$
\begin{aligned}
\left\|M_{T}^{N}\right\|_{2} & \leq \sum_{k=0}^{N-1}\left(1+\left\|c_{t_{k}^{N}}^{N}\right\|_{2}+\left\|V_{t_{k+1}^{N}}^{N}\right\|_{2}\right) \Delta_{k}^{N} \\
& \leq \sum_{k=0}^{N-1}\left(2+\left\|c_{t_{k}^{N}}^{N}\right\|_{2}^{2}+K_{2}\left[1+\|\xi\|_{2}+\|c\|_{2}^{2}\right]\right) \Delta_{k}^{N} \\
& \leq\left(2+K_{2}\left[1+\|\xi\|_{2}+\|c\|_{2}^{2}\right]\right) T+\sum_{k=0}^{N-1}\left\|c_{t_{k}^{N}}^{N}\right\|_{2}^{2} \Delta_{k}^{N} \\
& \leq\left(2+K_{2}\left[1+\|\xi\|_{2}+\|c\|_{2}^{2}\right]\right) T+\|c\|_{2}^{2}+1, N \geq N_{0}\left(c,\left\{c^{N}\right\}_{N \in \mathbb{N}}\right) .
\end{aligned}
$$

In combination with (12) this completes the proof.

Step 2. By (11) we have

$$
V_{t}^{N}=\mathbb{E}_{t}\left[\sum_{k=l}^{N-1} \Delta_{k}^{N} f\left(c_{t_{k}^{N}}^{N}, V_{t \vee t_{k}^{N}}^{N}\right)+\xi\right]+R_{t}^{1, N}+R_{t}^{2, N} \text { for } t \in\left[t_{l}^{N}, t_{l+1}^{N}\right)
$$

where $R^{2, N}=\left\{R_{t}^{2, N}\right\}_{t \in[0, T]}$ is given by

$$
R_{t}^{2, N} \triangleq \mathbb{E}_{t}\left[\sum_{k=l}^{N-1} \Delta_{k}^{N}\left\{f\left(c_{t_{k}^{N}}^{N}, \mathbb{E}_{t \vee t_{k}^{N}}\left[V_{t_{k+1}^{N}}^{N}\right]\right)-f\left(c_{t_{k}^{N}}^{N}, V_{t \vee t_{k}^{N}}^{N}\right)\right\}\right], t \in\left[t_{l}^{N}, t_{l+1}^{N}\right) .
$$

Lemma 5.2. There is a constant $K_{4}>0$ such that

$$
\left\|\sup _{t \in[0, T]}\left|R_{t}^{2, N}\right|\right\|_{2} \leq K_{4} \Delta^{N} \text { for all } N \geq N_{0}\left(c,\left\{c^{N}\right\}_{N \in \mathbb{N}}\right) .
$$

Proof. Condition (A2) implies

$$
\begin{aligned}
\left|R_{t}^{2, N}\right| & \leq \mathbb{E}_{t}\left[\sum_{k=l}^{N-1} \Delta_{k}^{N}\left|f\left(c_{t_{k}^{N}}^{N}, \mathbb{E}_{t \vee t_{k}^{N}}\left[V_{t_{k+1}^{N}}^{N}\right]\right)-f\left(c_{t_{k}^{N}}^{N}, V_{t \vee t_{k}^{N}}^{N}\right)\right|\right] \\
& \leq L \Delta^{N} \mathbb{E}_{t}\left[\sum_{k=l}^{N-1}\left|\mathbb{E}_{t \vee t_{k}^{N}}\left[V_{t_{k+1}^{N}}^{N}\right]-V_{t \vee t_{k}^{N}}^{N}\right|\right] \\
& =L \Delta^{N} \mathbb{E}_{t}\left[\sum_{k=l}^{N-1} \Delta_{k}^{N}\left|f\left(\Delta_{k}^{N}, c_{t_{k}^{N}}^{N}, \mathbb{E}_{t \vee t_{k}^{N}}\left[V_{t_{k+1}^{N}}^{N}\right]\right)\right|\right]
\end{aligned}
$$

where $t \in\left[t_{l}^{N}, t_{l+1}^{N}\right)$ and the identity uses (10). Moreover, (A3') yields

$$
\begin{aligned}
\left|R_{t}^{2, N}\right| & \leq K_{1} L \Delta^{N} \mathbb{E}_{t}\left[\sum_{k=l}^{N-1} \Delta_{k}^{N}\left[1+\left|c_{t_{k}^{N}}^{N}\right|+\left|\mathbb{E}_{t \vee t_{k}^{N}}\left[V_{t_{k+1}^{N}}^{N}\right]\right|\right]\right] \\
& \leq K_{1} L \Delta^{N} \mathbb{E}_{t}\left[\sum_{k=0}^{N-1} \Delta_{k}^{N}\left[1+\left|c_{t_{k}^{N}}^{N}\right|+\left|V_{t_{k+1}^{N}}^{N}\right|\right]\right] .
\end{aligned}
$$

Now the desired estimate follows exactly as in the proof of Lemma 5.1. 
For future reference, we rewrite (13) in integral form $\mathrm{as}^{9}$

$$
V_{t}^{N}=\mathbb{E}_{t}\left[\int_{t_{l}^{N}}^{T} f_{s}^{N, t} \mathrm{~d} s+\xi\right]+R_{t}^{1, N}+R_{t}^{2, N} \text { for } t \in\left[t_{l}^{N}, t_{l+1}^{N}\right)
$$

where $f^{N, t}=\left\{f_{s}^{N, t}\right\}_{s \in[0, T]}$ is given by

$$
f_{s}^{N, t} \triangleq f\left(c_{t_{k}^{N}}^{N}, V_{t \vee t_{k}^{N}}^{N}\right) \text { for } s \in\left[t_{k}^{N}, t_{k+1}^{N}\right), k=0, \ldots, N-1 .
$$

Step 3. By definition of $V$ we have

$$
V_{t}=\mathbb{E}_{t}\left[\int_{t}^{T} \tilde{f}_{s}^{N} \mathrm{~d} s+\xi\right]+R_{t}^{3, N} \text { for } t \in\left[t_{l}^{N}, t_{l+1}^{N}\right)
$$

where $\tilde{f}^{N}=\left\{\tilde{f}_{s}^{N}\right\}_{s \in[0, T]}$ is defined by

$$
\tilde{f}_{s}^{N} \triangleq f\left(c_{t_{k}^{N}}^{N}, V_{t_{k}^{N}}\right) \text { for } s \in\left[t_{k}^{N}, t_{k+1}^{N}\right), k=0, \ldots, N-1
$$

and where $R^{3, N}=\left\{R_{t}^{3, N}\right\}_{t \in[0, T]}$ is given by

$$
R_{t}^{3, N} \triangleq \mathbb{E}_{t}\left[\int_{t}^{T}\left\{f\left(c_{s}, V_{s}\right)-\tilde{f}_{s}^{N}\right\} \mathrm{d} s\right], t \in[0, T] .
$$

Lemma 5.3. We have

$$
\left\|\sup _{t \in[0, T]}\left|R_{t}^{3, N}\right|\right\|_{2} \rightarrow 0 \text { as } N \rightarrow \infty .
$$

Proof. For every $t \in[0, T]$ we have

$$
\left|R_{t}^{3, N}\right| \leq M_{t}^{N} \triangleq \mathbb{E}_{t}\left[\int_{0}^{T}\left|f\left(c_{s}, V_{s}\right)-\tilde{f}_{s}^{N}\right| \mathrm{d} s\right]
$$

with an $(\mathfrak{F}, \mathbb{P})$-martingale $M^{N}=\left\{M_{t}^{N}\right\}_{t \in[0, T]}$ satisfying $M_{T}^{N}=\int_{0}^{T}\left|f\left(c_{s}, V_{s}\right)-\tilde{f}_{s}^{N}\right| \mathrm{d} s$. Using Doob's L ${ }^{2}$-inequality and Cauchy's inequality we obtain

$$
\begin{aligned}
& \left\|\sup _{t \in[0, T]}\left|R_{t}^{3, N}\right|\right\|_{2} \leq\left\|\sup _{t \in[0, T]}\left|M_{t}^{N}\right|\right\|_{2} \leq 2\left\|M_{T}^{N}\right\|_{2} \\
= & 2\left\|\int_{0}^{T}\left|f\left(c_{s}, V_{s}\right)-\tilde{f}_{s}^{N}\right| \mathrm{d} s\right\|_{2} \leq 2 \sqrt{T} \mathbb{E}\left[\int_{0}^{T}\left|f\left(c_{s}, V_{s}\right)-\tilde{f}_{s}^{N}\right|^{2} \mathrm{~d} s\right]^{\frac{1}{2}} .
\end{aligned}
$$

By (2), the càdlàg property of $V,(\mathrm{~A} 3)$ and (A4) we have $\tilde{f}_{.}^{N} \rightarrow f(c ., V$.$) a.e. on$ $[0, T] \times \Omega$, so by (3), Proposition A.2, (A3') and dominated convergence we obtain

$$
\mathbb{E}\left[\int_{0}^{T}\left|f\left(c_{s}, V_{s}\right)-\tilde{f}_{s}^{N}\right|^{2} \mathrm{~d} s\right] \rightarrow 0 \text { as } N \rightarrow \infty .
$$

Remark. Lemma 5.3 is the only one of the error estimates established in this section that does not specify an explicit convergence rate. This cannot be expected unless we impose stricter assumptions (e.g., a Lipschitz condition) on the dependence of $W$ on $c$ and on the regularity of $c$ with respect to time. In this case, the literature on numerical solutions of BSDEs suggests that a convergence rate of $\sqrt{\Delta^{N}}$ could be expected in a jump-diffusion setting; see Zhang (2004), Bouchard and Elie (2008) or Bouchard, Elie, and Touzi (2009) and the references therein.

Step 4. By (15) we have

$$
\begin{aligned}
V_{t} & =\mathbb{E}_{t}\left[\int_{t_{l}^{N}}^{t_{l+1}^{N}} f\left(c_{t_{l}^{N}}^{N}, V_{t}\right) \mathrm{d} s+\int_{t_{l+1}^{N}}^{T} \tilde{f}_{s}^{N} \mathrm{~d} s+\xi\right]+R_{t}^{3, N}+R_{t}^{4, N} \\
& =\mathbb{E}_{t}\left[\int_{t_{l}^{N}}^{T} \hat{f}_{s}^{N, t} \mathrm{~d} s+\xi\right]+R_{t}^{3, N}+R_{t}^{4, N} \text { for } t \in\left[t_{l}^{N}, t_{l+1}^{N}\right)
\end{aligned}
$$

\footnotetext{
${ }^{9}$ The additional superindex $t$ indicates that $f^{N, t}$ depends explicitly on $t$.
} 
where $\hat{f}^{N, t}=\left\{\hat{f}_{s}^{N, t}\right\}_{s \in[0, T]}$ is given by

$$
\hat{f}_{s}^{N, t} \triangleq f\left(c_{t_{l}^{N}}^{N}, V_{t}\right), s \in\left[t_{l}^{N}, t_{l+1}^{N}\right), \quad \hat{f}_{s}^{N, t} \triangleq \tilde{f}_{s}^{N}, s \in\left[t_{l+1}^{N}, T\right]
$$

and $R^{4, N}=\left\{R_{t}^{4, N}\right\}_{t \in[0, T]}$ is given by

$$
R_{t}^{4, N} \triangleq-\int_{t_{l}^{N}}^{t} f\left(c_{t_{l}^{N}}^{N}, V_{t}\right) \mathrm{d} s+\mathbb{E}_{t}\left[\int_{t}^{t_{t+1}^{N}}\left\{\tilde{f}_{s}^{N}-f\left(c_{t_{l}^{N}}^{N}, V_{t}\right)\right\} \mathrm{d} s\right], t \in[0, T] .
$$

Lemma 5.4. There is a constant $K_{5}>0$ such that

$$
\left\|\sup _{t \in[0, T]}\left|R_{t}^{4, N}\right|\right\|_{2} \leq K_{5} \Delta^{N} \text { for all } N \in \mathbb{N} \text {. }
$$

Proof. By (A3') we have

$\left|\int_{t_{l}^{N}}^{t} f\left(c_{t_{l}^{N}}^{N}, V_{t}\right) \mathrm{d} s\right|,\left|\int_{t}^{t_{l+1}^{N}}\left\{\tilde{f}_{s}^{N}-f\left(c_{t_{l}^{N}}^{N}, V_{t}\right)\right\} \mathrm{d} s\right| \leq 2 \Delta^{N} K_{1}\left[1+\sup _{N \in \mathbb{N}, s \in[0, T]}\left|c_{s}^{N}\right|+\left|V_{s}\right|\right]$

so the desired conclusion follows immediately.

Gronwall-Bellman Argument. We are now in a position to complete the proof of Theorem 4.1. For every $t \in[0, T]$ we have

$$
\begin{aligned}
V_{t}^{N} & =\mathbb{E}_{t}\left[\int_{t_{l}^{N}}^{T} f_{s}^{N, t} \mathrm{~d} s+\xi\right]+R_{t}^{1, N}+R_{t}^{2, N} \\
V_{t} & =\mathbb{E}_{t}\left[\int_{t_{l}^{N}}^{T} \hat{f}_{s}^{N, t} \mathrm{~d} s+\xi\right]+R_{t}^{3, N}+R_{t}^{4, N}
\end{aligned}
$$

where $l$ chosen such that $t \in\left[t_{l}^{N}, t_{l+1}^{N}\right)$ and by Lemmas 5.1, 5.2, 5.3 and 5.4

$$
\left\|\sup _{t \in[0, T]}\left|R_{t}^{i, N}\right|\right\|_{2} \rightarrow 0 \text { as } N \rightarrow \infty \text { for } i=1,2,3,4 \text {. }
$$

Here $f^{N, t}=\left\{f_{s}^{N, t}\right\}_{s \in[0, T]}$ and $\hat{f}^{N, t}=\left\{\hat{f}_{s}^{N, t}\right\}_{s \in[0, T]}$ are given by

$$
\begin{array}{ll}
f_{s}^{N, t}=f\left(c_{t_{l}^{N}}^{N}, V_{t}^{N}\right), s \in\left[t_{l}^{N}, t_{l+1}^{N}\right), & f_{s}^{N, t}=f\left(c_{t_{k}^{N}}^{N}, V_{t_{k}^{N}}^{N}\right), s \in\left[t_{k}^{N}, t_{k+1}^{N}\right) \\
\hat{f}_{s}^{N, t}=f\left(c_{t_{l}^{N}}^{N}, V_{t}\right), s \in\left[t_{l}^{N}, t_{l+1}^{N}\right), & \hat{f}_{s}^{N, t}=\tilde{f}_{s}^{N}=f\left(c_{t_{k}^{N}}^{N}, V_{t_{k}^{N}}\right), s \in\left[t_{k}^{N}, t_{k+1}^{N}\right)
\end{array}
$$

for $k=l+1, \ldots, N-1$. Combining these results it follows that the process $D^{N}=$ $\left\{D_{t}^{N}\right\}_{t \in[0, T]}$ given by $D_{t}^{N} \triangleq V_{t}^{N}-V_{t}$ for $t \in[0, T]$ satisfies

$$
D_{t}^{N}=\mathbb{E}_{t}\left[\int_{t_{l}^{N}}^{T} r_{s}^{N, t} \mathrm{~d} s\right]+R_{t}^{N} \text { for } t \in\left[t_{l}^{N}, t_{l+1}^{N}\right)
$$

where $\left\|\sup _{t \in[0, T]}\left|R_{t}^{N}\right|\right\|_{2} \rightarrow 0$ as $N \rightarrow \infty$ and $r^{N, t}=\left\{r_{s}^{N, t}\right\}_{s \in[0, T]}$ is defined by $r_{s}^{N, t} \triangleq$ $f_{s}^{N, t}-\hat{f}_{s}^{N, t}$, i.e.

$$
\begin{aligned}
& r_{s}^{N, t}=f\left(c_{t_{l}^{N}}^{N}, V_{t}^{N}\right)-f\left(c_{t_{l}^{N}}^{N}, V_{t}\right), s \in\left[t_{l}^{N}, t_{l+1}^{N}\right), \\
& r_{s}^{N, t}=f\left(c_{t_{k}^{N}}^{N}, V_{t_{k}^{N}}^{N}\right)-f\left(c_{t_{k}^{N}}^{N}, V_{t_{k}^{N}}\right), s \in\left[t_{k}^{N}, t_{k+1}^{N}\right), k=l+1, \ldots, N-1 .
\end{aligned}
$$

The Lipschitz condition (A2) implies that

$$
\left|r_{s}^{N, t}\right| \leq L\left|D_{t}^{N}\right|, s \in\left[t_{l}^{N}, t_{l+1}^{N}\right) \text { and }\left|r_{s}^{N, t}\right| \leq L\left|D_{t_{k}^{N}}^{N}\right|, s \in\left[t_{k}^{N}, t_{k+1}^{N}\right)
$$

for $k=l+1, \ldots, N-1$. Therefore,

$$
\left|D_{t}^{N}\right| \leq L \mathbb{E}_{t}\left[\Delta_{l}^{N}\left|D_{t}^{N}\right|+\sum_{k=l+1}^{N} \Delta_{k}^{N}\left|D_{t_{k}^{N}}^{N}\right|\right]+\mathbb{E}_{t}\left[R^{N}\right]
$$

where $R^{N} \triangleq \sup _{t \in[0, T]}\left|R_{t}^{N}\right|$. If $N$ is sufficiently large so that $L \Delta^{N} \leq \frac{1}{2}$, we get

$$
\left|D_{t}^{N}\right| \leq 2 L \sum_{j=l+1}^{N} \Delta_{j}^{N} \mathbb{E}_{t}\left[\left|D_{t_{j}^{N}}^{N}\right|\right]+2 \mathbb{E}_{t}\left[R^{N}\right] .
$$


Analogously it follows that $\left|D_{t_{k}^{N}}^{N}\right| \leq 2 L \sum_{j=k+1}^{N} \Delta_{j}^{N} \mathbb{E}_{t_{k}^{N}}\left[\left|D_{t_{j}^{N}}^{N}\right|\right]+2 \mathbb{E}_{t_{k}^{N}}\left[R^{N}\right]$ for $k=l+$ $1, \ldots, N$ and therefore

$$
\mathbb{E}_{t}\left[\left|D_{t_{k}^{N}}^{N}\right|\right] \leq 2 L \sum_{j=k+1}^{N} \Delta_{j}^{N} \mathbb{E}_{t}\left[\left|D_{t_{j}^{N}}^{N}\right|\right]+2 \mathbb{E}_{t}\left[R^{N}\right], k=l+1, \ldots, N .
$$

Applying the discrete Gronwall Lemma A.3 to (18) and (19) yields $\left|D_{t}^{N}\right| \leq 2 \mathbb{E}_{t}\left[R^{N}\right] \mathrm{e}^{2 L T}$. Since $t \in[0, T]$ is arbitrary and $\left\|R^{N}\right\|_{2} \rightarrow 0$ as $N \rightarrow \infty$, we get

$$
\left\|\sup _{t \in[0, T]}\left|D_{t}^{N}\right|\right\|_{2} \rightarrow 0 \text { as } N \rightarrow \infty
$$

from Doob's $\mathrm{L}^{2}$-inequality. This completes the proof of Theorem 4.1.

\section{Convergence without (A1), (A2), (A3), (A4)}

In this section we provide a convergence result for bounded consumption plans. It applies to a wide class of aggregators that are relevant in applications but do not, in general, satisfy the conditions of Section 4.

A General Class of Aggregators. The discrete-time temporal aggregator is specified in terms of a strictly increasing function $g: C \subseteq[0, \infty) \rightarrow \mathbb{R}$ via

$$
W(\Delta, c, v) \triangleq g^{-1}(\delta \Delta g(c)+(1-\delta \Delta) g(v))
$$

where $\delta \in(0,1]$ models a subjective discount rate. With $\mathrm{V} \triangleq u(\mathrm{C})$ the corresponding normalized aggregator reads

$$
W:[0, T] \times \mathrm{C} \times \mathrm{V} \rightarrow \mathbb{R}, W(\Delta, c, v) \triangleq u \circ g^{-1}\left(\delta \Delta g(c)+(1-\delta \Delta) g \circ u^{-1}(v)\right)
$$

where $u$ is the function inducing the certainty equivalent via (5). The functions $g$ : $\mathrm{C} \rightarrow \mathbb{R}$ and $u: \quad \mathrm{C} \rightarrow \mathbb{R}$ are assumed to be of class $\mathrm{C}^{2}$ with

$$
g^{\prime}(c) \triangleq \frac{\partial g}{\partial c}(c)>0 \text { and } u^{\prime}(c) \triangleq \frac{\partial u}{\partial c}(c)>0, c \in \mathrm{C} .
$$

Note that (20) becomes additive for $u=g$, so discounted expected utility is a special case. By (8) the associated continuous-time aggregator is

$$
f: \mathbf{C} \times \mathrm{V} \rightarrow \mathbb{R}, \quad f(c, v)=\delta \frac{u^{\prime}\left(u^{-1}(v)\right)}{g^{\prime}\left(u^{-1}(v)\right)}\left[g(c)-g\left(u^{-1}(v)\right)\right] .
$$

A direct application of Theorem 4.1 may not be possible if (A1), (A2), (A3) and (A4) are not satisfied globally. ${ }^{10}$ In addition, $W(\Delta, c, \cdot)$ and $f(c, \cdot)$ may not be defined on all of $\mathbb{R}$. Below we present a suitable extension of Theorem 4.1 that applies in this context.

Example. The empirically relevant specification proposed by Epstein and Zin (1989) and Weil (1989) is obtained by choosing $\mathrm{C}=(0, \infty), g(c)=\frac{1}{1-\phi} c^{1-\phi}$ and $u(c)=$ $\frac{1}{1-\varrho} c^{1-\varrho}$. The parameters $\varrho>0$ and $\phi>0$ model relative risk aversion and the

\footnotetext{
${ }^{10}$ In this case, the continuous-time limit is not necessarily well-defined. For instance, if $u(c)=$ $g(c)=-c^{-2}$ and the consumption plan $(c, \xi)$ is given by $c_{t}=T-t, t \in[0, T]$ and $\xi=0$, then the recursive utility indices satisfy (see $(7))$

$$
\mathfrak{v}^{N}(c, \xi)=-\delta \sum_{k=0}^{N-1} \beta_{k}^{N} \frac{1}{\left(T-t_{k}^{N}\right)^{2}} \Delta_{k}^{N} \leq-\delta \beta_{N-1}^{N} \frac{1}{\Delta_{k}^{N}} \rightarrow-\infty \text { as } N \rightarrow \infty .
$$
}


inverse of the elasticity of intertemporal substitution. The corresponding normalized aggregator is given by

$$
W(\Delta, c, v)=\frac{1}{1-\varrho}\left[\delta \Delta c^{1-\phi}+(1-\delta \Delta)[(1-\varrho) v]^{\frac{1-\phi}{1-\varrho}}\right]^{\frac{1-\varrho}{1-\phi}}
$$

with $\mathrm{V}=(0, \infty)$ if $\varrho<1$ and $\mathrm{V}=(-\infty, 0)$ if $\varrho>1$. The associated continuous-time aggregator

$$
f(c, v)=\delta \frac{1-\varrho}{1-\phi} v\left[\left(\frac{c}{([1-\varrho] v)^{\frac{1}{1-\varrho}}}\right)^{1-\phi}-1\right]
$$

coincides with the standard parametrization used by Duffie and Epstein (1992), Fisher and Gilles (1998) and Wachter (2011), among others.

Convergence for Bounded Consumption Plans. Consider a consumption plan $c$ and an approximating sequence $\left\{c^{N}\right\}_{N \in \mathbb{N}}$ that take values in a closed and bounded subinterval $\mathrm{C}_{0} \subseteq \mathrm{C}$ and suppose that the terminal utility $\xi$ takes values in $\mathrm{V}_{0} \triangleq$ $u\left(\mathrm{C}_{0}\right) .{ }^{11}$ By $(21)$ the inverse functions $g^{-1}$ and $u^{-1}$ are of class $\mathrm{C}^{2}$ on $g\left(\mathrm{C}_{0}\right)$ and $u\left(\mathrm{C}_{0}\right)$, respectively, and it follows from the inverse mapping theorem that $W$ is of class $\mathrm{C}^{2}$ on $[0, T] \times \mathrm{C}_{0} \times \mathrm{V}_{0}$. Applying the mean value theorem twice yields

$$
f(\Delta, c, v)-f(c, v)=\frac{\partial W}{\partial \Delta}\left(\Delta^{\prime}, c, v\right)-\frac{\partial W}{\partial \Delta}(0, c, v)=\frac{\partial^{2} W}{\partial \Delta^{2}}\left(\Delta^{\prime \prime}, c, v\right) \Delta^{\prime}
$$

where $0<\Delta^{\prime \prime}<\Delta^{\prime}<\Delta$. Since $W$ is of class $\mathrm{C}^{2}$ it follows that $\frac{\partial^{2} W}{\partial \Delta^{2}}$ is bounded on $[0, T] \times \mathrm{C}_{0} \times \mathrm{V}_{0}$, so the restriction $W_{0}$ of $W$ to $[0, T] \times \mathrm{C}_{0} \times \mathrm{V}_{0}$ satisfies (A1). Similarly, (A2) holds for $W_{0}$. Finally, (A3) and (A4) are obvious.

Notice that for the aggregation $\left(\operatorname{Rec}^{N}\right)$ which determines $V^{N}=V^{N}\left(c^{N}\right)$ only the restriction $W_{0}$ is relevant. Indeed, one easily checks that $g^{-1}(\delta \Delta g(c)+(1-\delta \Delta) g(v)) \in$ $\mathrm{C}_{0}$ whenever $c, v \in \mathrm{C}_{0}$ and $\delta \Delta \leq 1$, so $V^{N}$ takes values in $\mathrm{V}_{0}$ for all but finitely many $N \in \mathbb{N}$. Hence we obtain

Theorem 6.1 (Convergence). Let $W$ be given by (20) where $g: C \rightarrow \mathbb{R}$ and $u: \mathrm{V} \rightarrow \mathbb{R}$ are $\mathrm{C}^{2}$ functions that satisfy (21) and suppose that $\xi$ takes values in a bounded closed subset of $\mathrm{V}$. If $c \in \mathcal{A}$ is a consumption plan and $\left\{c^{N}\right\}_{N \in \mathbb{N}}$ is an approximating sequence that take values in bounded closed subintervals of $\mathrm{C}$, then

$$
\left\|\sup _{t \in[0, T]}\left|V_{t}^{N}-V_{t}\right|\right\|_{2} \rightarrow 0 \text { and in particular } \mathfrak{v}^{N}\left(c^{N}\right) \rightarrow \mathfrak{v}(c) \text { as } N \rightarrow \infty .
$$

Here $\mathfrak{v}^{N}$ and $\mathfrak{v}$ denote, respectively, the recursive utility index on $\pi^{N}$ and the stochastic differential utility index with aggregator $f$ given by (22), and $V^{N}$ and $V$ are the associated continuation value processes.

Proof. Choose a closed interval $\mathrm{C}_{0} \subseteq \mathrm{C}$ such that $c$ and $c^{N}$ take values in $\mathrm{C}_{0}$ for $N \in \mathbb{N}$ and $\xi$ takes values in $\mathrm{V}_{0} \triangleq u\left(\mathrm{C}_{0}\right)$. Let $W_{0}$ denote the restriction of $W$ to $[0, T] \times \mathrm{C}_{0} \times \mathrm{V}_{0}$; by the preceding discussion $W_{0}$ satisfies (A1), (A2), (A3) and (A4) on $[0, T] \times \mathrm{C}_{0} \times \mathrm{V}_{0}$. Now select an extension $\bar{W}_{0}$ of $W_{0}$ to $[0, T] \times \mathbf{C} \times \mathbb{R}$ in such a way that (A1), (A2), (A3) and (A4) hold for $\bar{W}_{0}$ on $[0, T] \times \mathbf{C} \times \mathbb{R}$. Now we can apply Theorem 4.1 with the aggregator $\bar{W}_{0}$. As observed above, the process $V^{N}=V^{N}\left(c^{N}\right)$ takes values in $\mathrm{V}_{0}$ for all but finitely many $N \in \mathbb{N}$ and, in particular, does not depend on the choice of the extension $\bar{W}_{0}$; since $\left\|V_{t}^{N}-V_{t}\right\| \rightarrow 0$ as $N \rightarrow \infty$ it follows that $V=V(c)$ cannot depend on the choice of $\bar{W}_{0}$ either. The claim now follows from Theorem 4.1.

\footnotetext{
${ }^{11}$ This is consistent: The corresponding monetary payoff $X=u^{-1}(\xi)$ is $\mathrm{C}_{0}$-valued.
} 
Remark. For Epstein-Zin-Weil utility, Theorem 6.1 requires consumption to be bounded from below by a positive constant. This may not be satisfied in specific applications as it interferes with the homotheticity of the Epstein-Zin aggregator.

\section{Beyond Kreps-Porteus Certainty Equivalents}

Recently certainty equivalents outside the Kreps-Porteus specification have become important in the modeling of ambiguity, source-dependent risk aversion, and further behavioral aspects of decisions under risk. In this section we provide a basis for research on recursive utility with nonstandard risk preferences: We establish a sufficient criterion for a general certainty equivalent to lead to stochastic differential utility in the continuous-time limit.

Normalization. Consider a consumption plan $c \in \mathcal{A}$, an approximating sequence $\left\{c^{N}\right\}_{N \in \mathbb{N}}$ and the associated backward recursion (4) that defines recursive utility,

$$
V_{t_{k}^{N}}^{N}=W\left(\Delta_{k}^{N}, c_{t_{k}^{N}}^{N}, \mathfrak{m}_{t_{k}^{N}}\left(V_{t_{k+1}^{N}}^{N}\right)\right), k=0, \ldots, N-1, \quad V_{t_{N}^{N}}^{N}=X .
$$

Here $\left\{\mathfrak{m}_{t}\right\}_{t \in[0, T]}$ is a family of abstract time- $t$ certainty equivalents (not necessarily of Kreps-Porteus type). As in Section 3 the normalization $v \mapsto \tilde{v} \triangleq u(v)$ with $u$ strictly increasing leads to the ordinally equivalent representation

$$
\tilde{V}_{t_{k}^{N}}^{N}=\tilde{W}\left(\Delta_{k}^{N}, c_{t_{k}^{N}}^{N}, \tilde{\mathfrak{m}}_{t_{k}^{N}}\left(\tilde{V}_{t_{k+1}^{N}}^{N}\right)\right), k=0, \ldots, N-1, \quad \tilde{V}_{t_{N}^{N}}^{N}=\xi
$$

where $\xi \triangleq u(X)$, the normalized time-t certainty equivalent $\tilde{\mathfrak{m}}_{t}$ is given by

$$
\tilde{\mathfrak{m}}_{t}(\tilde{V}) \triangleq u\left(\mathfrak{m}_{t}\left(u^{-1}(\tilde{V})\right)\right)
$$

and $\tilde{W}(\Delta, c, \tilde{v}) \triangleq u \circ W\left(\Delta, c, u^{-1}(\tilde{v})\right)$.

While, in general, it may not be possible to achieve an exact normalization as in Section 3 for the Kreps-Porteus case, motivated by the analysis in Skiadas (2013) we consider the case when an approximate normalization is available:

$$
\tilde{\mathfrak{m}}_{t}(\tilde{V})=\mathbb{E}_{t}^{\mathbb{Q}}[\tilde{V}]+R_{t}
$$

with a probability measure $\mathbb{Q}$ and a "small" error $R_{t}$. In the following we establish a general convergence criterion based on an approximation of this form.

Theorem 7.1 (Convergence with Approximate Normalization). Consider a normalized aggregator $W=\tilde{W}$ as in (23) that satisfies (A1), (A2), (A3), (A4). Let $\mathbb{Q}$ be an equivalent probability measure such that $\left\{c^{N}\right\}_{N \in \mathbb{N}}$ is also an approximating sequence for $c$ with respect to $\mathbb{Q}$ and $\mathbb{E}^{\mathbb{Q}}\left[|\xi|^{2}\right]<\infty$. If the normalized certainty equivalents (24) satisfy

$$
\mathfrak{m}_{t_{k}^{N}}\left(V_{t_{k+1}^{N}}^{N}\right)=\mathbb{E}_{t_{k}^{N}}^{\mathbb{Q}}\left[V_{t_{k+1}^{N}}^{N}\right]+R_{t_{k}^{N}}^{N} \text { for } k=0, \ldots, N-1
$$

where $\sum_{k=1}^{N-1}\left\|R_{t_{k}^{N}}^{N}\right\|_{1, \mathbb{Q}} \rightarrow 0$ as $N \rightarrow \infty$, then we have $\mathfrak{v}^{N}\left(c^{N}\right) \rightarrow \mathfrak{v}(c)$ as $N \rightarrow \infty$ where $\mathfrak{v}(c)=V_{0}$ and $V$ denotes the stochastic differential utility process

$$
V_{t}=\mathbb{E}_{t}^{\mathbb{Q}}\left[\int_{t}^{T} f\left(c_{s}, V_{s}\right) \mathrm{d} s+\xi\right], t \in[0, T] \text { with } f(c, v)=\frac{\partial W}{\partial \Delta}(0, c, v) .
$$


Proof. By (25) we can rewrite the recursion (23) as

$$
V_{t_{k}^{N}}^{N}=\mathbb{E}_{t_{k}^{N}}^{\mathbb{Q}}\left[\Delta_{k}^{N} f\left(\Delta_{k}^{N}, c_{t_{k}^{N}}^{N}, \mathfrak{m}_{t_{k}^{N}}\left(V_{t_{k+1}^{N}}^{N}\right)\right)+V_{t_{k+1}^{N}}^{N}+R_{t_{k}^{N}}^{N}\right], k=0, \ldots, N-1
$$

and similarly as in Section 5 iterating this leads to

$$
V_{t_{l}^{N}}^{N}=\mathbb{E}_{t_{l}^{N}}^{\mathbb{Q}}\left[\sum_{k=l}^{N-1} \Delta_{k}^{N} f\left(\Delta_{k}^{N}, c_{t_{k}^{N}}^{N}, \mathfrak{m}_{t_{k}^{N}}\left(V_{t_{k+1}^{N}}^{N}\right)\right)+\xi+\sum_{k=l}^{N-1} R_{t_{k}^{N}}^{N}\right] .
$$

Now Theorem 4.1 implies $\max _{k=0, \ldots, N}\left|\tilde{V}_{t_{k}^{N}}^{N}-V_{t_{k}^{N}}\right| \rightarrow 0$ in $\mathrm{L}^{2}(\mathbb{Q})$ as $N \rightarrow \infty$ where

$$
\tilde{V}_{t_{l}^{N}}^{N} \triangleq \mathbb{E}_{t_{l}^{N}}^{\mathbb{Q}}\left[\sum_{k=l}^{N-1} \Delta_{k}^{N} f\left(\Delta_{k}^{N}, c_{t_{k}^{N}}^{N}, \mathbb{E}_{t_{k}^{N}}^{\mathbb{Q}}\left[\tilde{V}_{t_{k+1}^{N}}^{N}\right]\right)+\xi\right], l=0, \ldots, N .
$$

Thus it suffices to show that

$$
\max _{k=0, \ldots, N}\left\|V_{t_{k}^{N}}^{N}-\tilde{V}_{t_{k}^{N}}^{N}\right\|_{1, \mathbb{Q}} \rightarrow 0 \text { as } N \rightarrow \infty .
$$

To verify this note that, by (A1') and (A2), $|f(\Delta, c, v)-f(\Delta, c, w)| \leq L|v-w|+$ $2 h(\Delta)[1+|c|+|v|+|w|]$, so by (25) and the conditional triangle inequality

$$
\begin{aligned}
\left|V_{t_{l}^{N}}^{N}-\tilde{V}_{t_{l}^{N}}^{N}\right| \leq & \mathbb{E}_{t_{l}^{N}}^{\mathbb{Q}}\left[\sum_{k=l}^{N-1} \Delta_{k}^{N} L\left|V_{t_{k+1}^{N}}^{N}-\tilde{V}_{t_{k+1}^{N}}^{N}\right|\right]+\mathbb{E}_{t_{l}^{N}}^{\mathbb{Q}}\left[\sum_{k=l}^{N-1}\left(1+\Delta_{k}^{N} L\right)\left|R_{t_{k}^{N}}^{N}\right|\right] \\
& +\mathbb{E}_{t_{l}^{N}}^{\mathbb{Q}}\left[2 h\left(\Delta_{k}^{N}\right) \sum_{k=l}^{N-1} \Delta_{k}^{N}\left(\left|c_{t_{k}^{N}}^{N}\right|+\left|V_{t_{k+1}^{N}}^{N}\right|+\left|\tilde{V}_{t_{k+1}^{N}}^{N}\right|\right)\right], l=0, \ldots, N .
\end{aligned}
$$

We have $\sup _{N \in \mathbb{N}} \max _{k=0, \ldots, N}\left\|c_{t_{k}^{N}}^{N}\right\|_{2, \mathbb{Q}}<\infty$ since $\left\{c^{N}\right\}_{N \in \mathbb{N}}$ is an approximating sequence under $\mathbb{Q}$. As in Proposition A.1 we can use (A3') to establish the a priori estimate $\sup _{N \in \mathbb{N}} \max _{k=0, \ldots, N}\left\|V_{t_{k}^{N}}^{N}\right\|_{1, \mathbb{Q}}<\infty$. By the first part of the proof, we have $\sup _{N \in \mathbb{N}} \max _{k=0, \ldots, N}\left\|\tilde{V}_{t_{k}^{N}}^{N}\right\|_{2, \mathbb{Q}}<\infty$ and thus it follows that

$\left\|V_{t_{l}^{N}}^{N}-\tilde{V}_{t_{l}^{N}}^{N}\right\|_{1, \mathbb{Q}} \leq \sum_{k=l}^{N-1} \Delta_{k}^{N} L\left\|V_{t_{k+1}^{N}}^{N}-\tilde{V}_{t_{k+1}^{N}}^{N}\right\|_{1, \mathbb{Q}}+\left(1+\Delta^{N} L\right) \sum_{k=1}^{N-1}\left\|R_{t_{k}^{N}}^{N}\right\|_{1, \mathbb{Q}}+K h\left(\Delta^{N}\right)$

with a constant $K>0$. Now the Gronwall Lemma A.3 yields the result.

Example: Drift Uncertainty. Suppose $W$ is a $(\mathbb{P}, \mathfrak{F})$-Wiener process and $\left\{\mu_{t}^{i}(s)\right\}_{s \in[t, T]}$ is a family of progressively measurable processes for each $t \in[0, T]$ and $i=1, \ldots, d$. For $t, t+\Delta \in[0, T]$ define the drift-adjusted probability $\mathbb{Q}_{t, t+\Delta}^{i}$ on $\mathfrak{F}_{t+\Delta}$ by

$$
\frac{\mathrm{d} \mathbb{Q}_{t, t+\Delta}^{i}}{\mathrm{~d} \mathbb{P}}=\exp \left\{\int_{t}^{t+\Delta} \mu_{t}^{i}(s) \mathrm{d} W(s)-\frac{1}{2} \int_{t}^{t+\Delta}\left|\mu_{t}^{i}(s)\right|^{2} \mathrm{~d} s\right\}
$$

and assume the normalized time- $t$ certainty equivalent is given by

$$
\mathfrak{m}_{t}(V)=\Phi_{t}\left(\mathbb{E}_{t, t+\Delta}^{i}[V]: i=1, \ldots, d\right) \text { for } V \in \mathfrak{F}_{t+\Delta} .
$$

Here $\mathbb{E}_{t, t+\Delta}^{i}$ denotes $\mathbb{Q}_{t, t+\Delta^{-}}^{i}$-conditional expectation given $\mathfrak{F}_{t}$, and for each $t \in[0, T]$ the random function $\Phi_{t}: \mathbb{R}^{d} \rightarrow \mathbb{R}$ is $\mathfrak{F}_{t}$-measurable, satisfies $\Phi_{t}(x, \ldots, x)=x$ for all $x \in \mathbb{R}$ and there is a constant $L>0$ such that

$$
\left|\Phi_{t}(x)-\Phi_{t}(y)\right| \leq L|x-y| \text { for } x, y \in \mathbb{R}^{d} .
$$

Examples include the robust specification $\Phi_{t}(x)=\min (x)$ and the second-order expectation $\Phi_{t}(x)=\sum_{i=1}^{d} \alpha_{t}^{i} x^{i}$ with adapted probability weights $\alpha^{i}=\left\{\alpha_{t}^{i}\right\}_{t \in[0, T]}$.

In this setting, if there are a progressive process $\mu^{0}=\left\{\mu^{0}(t)\right\}_{t \in[0, T]}$ and a continuous increasing function $h:[0, T] \rightarrow[0, \infty)$ with $h(0)=0$ such that

$$
\left|\mu_{t}^{i}(s)-\mu^{0}(t)\right| \leq h(s-t) \sqrt{s-t} \text { for } s \geq t \text { and } i=1, \ldots, d
$$


then condition (25) in Theorem 7.1 is satisfied with $\mathbb{Q}$ defined via

$$
\frac{\mathrm{d} \mathbb{Q}}{\mathrm{d} \mathbb{P}}=\exp \left\{\int_{0}^{T} \mu^{0}(s) \mathrm{d} W(s)-\frac{1}{2} \int_{0}^{T}\left|\mu^{0}(s)\right|^{2} \mathrm{~d} s\right\}
$$

provided the integrability condition $\sup _{N \in \mathbb{N}} \max _{k=0, \ldots, N}\left\|V_{t_{k}^{N}}^{N}\right\|_{2, \mathbb{Q}}<\infty$ holds. A proof is provided in Appendix B.

\section{Conclusion}

Summary. The convergence results of this paper rigorously establish stochastic differential utility as the continuous-time limit of Kreps-Porteus recursive utility. Thus they close an important gap in the literature and justify the use of stochastic differential utility in continuous-time models in macroeconomics and finance.

Directions for Future Research. The extension of our analysis to the case of an infinite time horizon is an interesting subject for further study. It would also be interesting to establish a more general convergence result for the special case of EpsteinZin-Weil utility that also covers unbounded consumption rate processes, based on the analysis of Schroder and Skiadas (1999). In a Brownian setting, one can further extend the analysis of this paper to the notion of generalized stochastic differential utility analyzed by Lazrak (2004).

In Section 7 we have provided a basis for the analysis of preferences with nonstandard certainty equivalents. These results can be extended and generalized, in particular to specifications when an approximate normalization is not available. Finally, we expect our results to be relevant for the numerical evaluation of stochastic differential utility. A detailed analysis of these challenging aspects is left for future research.

\section{Appendix A. A Priori Estimates and Gronwall Inequality}

Throughout this appendix, let $c \in \mathcal{A}$ be a consumption plan with an associated approximating sequence $\left\{c^{N}\right\}_{N \in \mathbb{N}}$, let $\mathbb{E}\left[|\xi|^{2}\right]<\infty$ and let the aggregator $W$ satisfy (A1), (A2), (A3) and (A4). By (2), (3) and dominated convergence we have

$$
\left\|c^{N}-c\right\|_{2}^{2} \rightarrow 0 \text { and in particular }\left\|c^{N}\right\|_{2}^{2}=\sum_{k=0}^{N-1} \Delta_{k}^{N}\left\|c_{t_{k}^{N}}^{N}\right\|_{2}^{2} \rightarrow\|c\|_{2}^{2} \text { as } N \rightarrow \infty
$$

where $\|c\|_{2}^{2} \triangleq \mathbb{E}\left[\int_{0}^{T} c_{t}^{2} \mathrm{~d} t\right]$. Hence there exists $N_{0}\left(c,\left\{c^{N}\right\}_{N \in \mathbb{N}}\right) \in \mathbb{N}$ such that

$$
\sum_{k=0}^{N-1} \Delta_{k}^{N}\left\|c_{t_{k}^{N}}^{N}\right\|_{2}^{2} \leq 1+\|c\|_{2}^{2} \text { for all } N \geq N_{0}\left(c,\left\{c^{N}\right\}_{N \in \mathbb{N}}\right) .
$$

The following $\mathrm{L}^{2}$-bounds are used in the proof of Theorem 4.1.

Proposition A.1 (A Priori Estimate for Recursive Utility). For every approximating sequence $\left\{c^{N}\right\}_{N \in \mathbb{N}}$ of $c \in \mathcal{A}$ there is a constant $K_{2}>0$ such that

$$
\left\|\sup _{t \in[0, T]}\left|V_{t}^{N}\left(c^{N}\right)\right|\right\|_{2} \leq K_{2}\left[1+\|\xi\|_{2}+\|c\|_{2}^{2}\right] \text { for all } N \geq N_{0}\left(c,\left\{c^{N}\right\}_{N \in \mathbb{N}}\right) .
$$


Proof. Consider (10) and use the conditional triangle inequality and (A3') to get

$$
\begin{aligned}
\left|V_{t}^{N}\right| & \leq \mathbb{E}_{t}\left[\left|V_{t_{k+1}^{N}}^{N}\right|\right]+\Delta_{k}^{N} \mathbb{E}_{t}\left[\left|f\left(\Delta_{k}^{N}, c_{t_{k}^{N}}^{N}, \mathbb{E}_{t}\left[V_{t_{k+1}^{N}}^{N}\right]\right)\right|\right] \\
& \leq \mathbb{E}_{t}\left[\left|V_{t_{k+1}^{N}}^{N}\right|\right]+\Delta_{k}^{N} K_{1} \mathbb{E}_{t}\left[1+\left|c_{t_{k}^{N}}^{N}\right|+\left|\mathbb{E}_{t}\left[V_{t_{k+1}^{N}}^{N}\right]\right|\right] \\
& \leq \mathbb{E}_{t}\left[\left(1+K_{1} \Delta_{k}^{N}\right)\left|V_{t_{k+1}^{N}}^{N}\right|+K_{1} \Delta_{k}^{N}\left(1+\left|c_{t_{k}^{N}}^{N}\right|\right)\right] \text { for } t \in\left[t_{k}^{N}, t_{k+1}^{N}\right) .
\end{aligned}
$$

Iterating this estimate and using the fact that $1+x \leq e^{x}$ for $x \in \mathbb{R}$ we find

$$
\begin{aligned}
\left|V_{t}^{N}\right| & \leq \mathbb{E}_{t}\left[\prod_{k=l}^{N-1}\left(1+K_{1} \Delta_{k}^{N}\right)|\xi|+\sum_{k=l}^{N-1} \prod_{j=l}^{k-1}\left(1+K_{1} \Delta_{j}^{N}\right) K_{1} \Delta_{k}^{N}\left(1+\left|c_{t_{k}^{N}}^{N}\right|\right)\right] \\
& \leq M_{t}^{N} \triangleq \mathbb{E}_{t}\left[\mathrm{e}^{K_{1} T}|\xi|+K_{1} \mathrm{e}^{K_{1} T} \sum_{k=0}^{N-1} \Delta_{k}^{N}\left(1+\left|c_{t_{k}^{N}}^{N}\right|\right)\right], t \in\left[t_{l}^{N}, t_{l+1}^{N}\right)
\end{aligned}
$$

where the process $M^{N}=\left\{M_{t}^{N}\right\}_{t \in[0, T]}$ is an $(\mathfrak{F}, \mathbb{P})$-martingale with

$$
\begin{aligned}
\left\|M_{T}^{N}\right\|_{2} & \leq \mathrm{e}^{K_{1} T}\|\xi\|_{2}+K_{1} \mathrm{e}^{K_{1} T} \sum_{k=0}^{N-1} \Delta_{k}^{N}\left(1+\left\|c_{t_{k}^{N}}^{N}\right\|_{2}\right) \\
& \leq \mathrm{e}^{K_{1} T}\|\xi\|_{2}+K_{1} \mathrm{e}^{K_{1} T} \sum_{k=0}^{N-1} \Delta_{k}^{N}\left(2+\left\|c_{t_{k}^{N}}^{N}\right\|_{2}^{2}\right) \\
& \leq \mathrm{e}^{K_{1} T}\|\xi\|_{2}+K_{1} \mathrm{e}^{K_{1} T}\left(2 T+\|c\|_{2}^{2}+1\right), N \geq N_{0}\left(c,\left\{c^{N}\right\}_{N \in \mathbb{N}}\right) .
\end{aligned}
$$

Here we have used (27) and the fact that $x \leq 1+x^{2}$ for all $x \in \mathbb{R}$. Since

$$
\left\|\sup _{t \in[0, T]}\left|V_{t}^{N}\right|\right\|_{2} \leq\left\|\sup _{t \in[0, T]} M_{t}^{N}\right\|_{2} \leq 2\left\|M_{T}^{N}\right\|_{2}
$$

by (28) and Doob's L $\mathrm{L}^{2}$-inequality, this completes the proof.

Proposition A.2 (A Priori Estimate for SDU). For every $c \in \mathcal{A}$ there exists a unique càdlàg process $V=V(c)=\left\{V_{t}(c)\right\}_{t \in[0, T]}$ with $\mathbb{E}\left[\sup _{t \in[0, T]}\left|V_{t}(c)\right|^{2}\right]<\infty$ such that (SDU) is satisfied. Moreover, there is a constant $K_{2}^{\prime}>0$ such that

$$
\left\|\sup _{t \in[0, T]}\left|V_{t}(c)\right|\right\|_{2} \leq K_{2}^{\prime}\left[1+\|\xi\|_{2}+\|c\|_{2}^{2}\right] \text { for every } c \in \mathcal{A} \text {. }
$$

Proof. By (A2) and (A3) the function $f$ satisfies a Lipschitz and linear growth condition. Hence the claim follows from well-known results on BSDEs; see, e.g., Duffie and Epstein (1992) or Antonelli (1993).

Lemma A.3 (Discrete Gronwall Inequality). Let $\left\{d_{k}\right\}_{k=0, \ldots, N}$ satisfy

$$
d_{k} \leq \varepsilon+\sum_{j=k+1}^{N} \alpha_{j} d_{j} \text { for } k=0, \ldots, N
$$

where $\left\{\alpha_{k}\right\}_{k=0, \ldots, N}$ is a sequence of non-negative numbers and $\varepsilon \geq 0$. Then

$$
d_{k} \leq \varepsilon \prod_{j=k+1}^{N}\left(1+\alpha_{j}\right) \leq \varepsilon \mathrm{e}^{\sum_{j=k+1}^{N} \alpha_{j}} \text { for all } k=0, \ldots, N .
$$

Proof. Set $x_{k} \triangleq \varepsilon+\sum_{j=k+1}^{N} \alpha_{j} x_{j}, k=N, \ldots, 0$. By induction

$$
x_{k}=\varepsilon \prod_{j=k+1}^{N}\left(1+\alpha_{j}\right), k=0, \ldots, N .
$$

Indeed, this is clear for $k=N$; if (29) holds for $j=k+1, \ldots, N$, then

$$
\begin{aligned}
x_{k} & =\varepsilon+\sum_{j=k+1}^{N} \alpha_{j} x_{j}=\varepsilon+\sum_{j=k+1}^{N} \alpha_{j} \varepsilon \prod_{i=j+1}^{N}\left(1+\alpha_{i}\right) \\
& =\varepsilon\left[1+\sum_{j=k+1}^{N} \prod_{i=j}^{N}\left(1+\alpha_{i}\right)-\prod_{i=j+1}^{N}\left(1+\alpha_{i}\right)\right]=\varepsilon \prod_{i=k+1}^{N}\left(1+\alpha_{i}\right) .
\end{aligned}
$$

Another induction argument yields $d_{k} \leq x_{k}$ for $k=0, \ldots, N$. 


\section{Appendix B. Proof of (25) for Drift Ambiguity}

We assume without loss that $\mu^{0}=0$. By definition of $\mathbb{Q}_{t, t+\Delta}^{i}$ we have $\mathbb{E}_{t, t+\Delta}^{i}[V]=$ $\mathbb{E}_{t}^{\mathbb{Q}}[V]+\mathbb{E}_{t}^{\mathbb{Q}}\left[Z_{t, t+\Delta}^{i} V\right]$ with $Z_{t, t+\Delta}^{i} \triangleq \frac{\mathrm{dQ}_{t, t+\Delta}^{i}}{\mathrm{~d} \mathbb{Q}}-1$. The Lipschitz property of $\Phi_{t}$ yields

$$
\begin{aligned}
\left|\mathfrak{m}_{t}(V)-\mathbb{E}_{t}^{\mathbb{Q}}[V]\right| & =\left|\Phi_{t}\left(\mathbb{E}_{t}^{\mathbb{Q}}[V]+\mathbb{E}_{t}^{\mathbb{Q}}\left[Z_{t, t+\Delta}^{i} V\right]: i=1, \ldots, d\right)-\mathbb{E}_{t}^{\mathbb{Q}}[V]\right| \\
& \leq L \max _{i=1, \ldots, d} \mathbb{E}_{t}^{\mathbb{Q}}\left[Z_{t, t+\Delta}^{i}|V|\right] \text { for } V \in \mathfrak{F}_{t+\Delta}
\end{aligned}
$$

and therefore we obtain

$$
\left\|R_{t_{k}^{N}}^{N}\right\|_{1, \mathbb{Q}}=\mathbb{E}^{\mathbb{Q}}\left[\left|\mathfrak{m}_{t_{k}^{N}}\left(V_{t_{k+1}^{N}}^{N}\right)-\mathbb{E}_{t_{k}^{N}}^{\mathbb{Q}}\left[V_{t_{k+1}^{N}}^{N}\right]\right|\right] \leq L \max _{i=1, \ldots, d} \mathbb{E}^{\mathbb{Q}}\left[Z_{t_{k}^{N}, t_{k+1}^{N}}^{i}\left|V_{t_{k+1}^{N}}^{N}\right|\right] .
$$

By Cauchy's inequality it follows that

$$
\sum_{k=1}^{N-1}\left\|R_{t_{k}^{N}}^{N}\right\|_{1, \mathbb{Q}} \leq L^{\prime} \sum_{k=1}^{N-1} \max _{i=1, \ldots, d}\left\|Z_{t_{k}^{N}, t_{k+1}^{N}}^{i}\right\|_{2, \mathbb{Q}}
$$

where $L^{\prime} \triangleq L \sup _{N \in \mathbb{N}} \max _{k=0, \ldots, N}\left\|V_{t_{k}^{N}}^{N}\right\|_{2, \mathbb{Q}}$. With $\bar{\mu}_{t}^{i} \triangleq 2 \mu_{t}^{i}$ we have from (26)

$$
\begin{aligned}
\left\|Z_{t, t+\Delta}^{i}\right\|_{2, \mathbb{Q}}^{2} & =\mathbb{E}^{\mathbb{Q}}\left[\left(\exp \left\{\int_{t}^{t+\Delta} \mu_{t}^{i}(s) \mathrm{d} W(s)-\frac{1}{2} \int_{t}^{t+\Delta}\left|\mu_{t}^{i}(s)\right|^{2} \mathrm{~d} s\right\}-1\right)^{2}\right] \\
& =\mathbb{E}^{\mathbb{Q}}\left[\exp \left\{\int_{t}^{t+\Delta} \bar{\mu}_{t}^{i}(s) \mathrm{d} W(s)-\frac{1}{2} \int_{t}^{t+\Delta}\left|\bar{\mu}_{t}^{i}(s)\right|^{2} \mathrm{~d} s+\int_{t}^{t+\Delta}\left|\mu_{t}^{i}(s)\right|^{2} \mathrm{~d} s\right\}\right]-1 \\
& \leq \mathbb{E}^{\mathbb{Q}}\left[\exp \left\{\int_{t}^{t+\Delta} \bar{\mu}_{t}^{i}(s) \mathrm{d} W(s)-\frac{1}{2} \int_{t}^{t+\Delta}\left|\bar{\mu}_{t}^{i}(s)\right|^{2} \mathrm{~d} s+\frac{1}{2} h(\Delta)^{2} \Delta^{2}\right\}\right]-1 \\
& =\mathrm{e}^{\frac{1}{2} h(\Delta)^{2} \Delta^{2}}-1 \leq h(\Delta)^{2} \Delta^{2} \text { for sufficiently small } \Delta>0 .
\end{aligned}
$$

Hence the assertion follows from (30) and the estimate

$$
\sum_{k=1}^{N-1} \max _{i=1, \ldots, d}\left\|Z_{t_{k}^{N}, t_{k+1}^{N}}^{i}\right\|_{2, \mathbb{Q}} \leq \sum_{k=1}^{N-1} h\left(\Delta_{k}^{N}\right) \Delta_{k}^{N} \leq h\left(\Delta^{N}\right) T \rightarrow 0 \text { as } N \rightarrow \infty .
$$

\section{REFERENCES}

Antonelli, Fabio, 1993, Backward-forward stochastic differential equations, Annals of Applied Probability 3, 777-793.

— , 1996, Stability of backward stochastic differential equations, Stochastic Processes and Their Applications 62, 103-114.

Bansal, Ravi, and Amir Yaron, 2004, Risks for the long run: A potential resolution of asset pricing puzzles, Journal of Finance 59, 1481-1509.

Borovička, Jaroslav, Lars Peter Hansen, Mark Hendricks, and José Scheinkman, 2011, Risk-price dynamics, Journal of Financial Econometrics 9, 3-65.

Bouchard, Bruno, and Romuald Elie, 2008, Discrete-time approximation of decoupled forward-backward SDE with jumps, Stochastic Processes and Their Applications $118,53-75$.

— , and Nizar Touzi, 2009, Discrete-time approximation of BSDEs and probabilistic schemes for fully nonlinear PDEs, Radon Series in Computational and Applied Mathematics 8, 1-34.

Bouchard, Bruno, and Nizar Touzi, 2004, Discrete-time approximation and Monte Carlo simulation of backward stochastic differential equations, Stochastic Processes and Their Applications 111, 175-206. 
Coquet, François, Vigirdas Mackevičius, and Jean Mémin, 1998, Stability in D of martingales and backward equations under discretization of filtration, Stochastic Processes and Their Applications 75, 235-248.

Cox, John C., Stephen A. Ross, and Mark Rubinstein, 1979, Option pricing: A simplified approach, Journal of Financial Economics 7, 229-263.

Duffie, Darrell, and Larry G. Epstein, 1992, Stochastic differential utility, Econometrica $60,353-394$.

— , 1992b, Asset pricing with stochastic differential utility, Review of Financial Studies 5, 411-436.

Duffie, Darrell, and Philip Protter, 1992, From discrete- to continuous-time finance: Weak convergence of the financial gain process, Mathematical Finance 2, 1-15.

El Karoui, Nicole, Shige Peng, and Marie-Claire Quenez, 1997, Backward stochastic differential equations in finance, Mathematical Finance 7, 1-71.

Epstein, Larry G., 1987, The global stability of efficient intertemporal allocations, Econometrica 55, 329-358.

- and Stanley E. Zin, 1989, Substitution, risk aversion and the temporal behavior of consumption and asset returns: A theoretical framework, Econometrica 57, 937-969.

Fisher, Mark, and Christian Gilles, 1998, Consumption and asset prices with recursive preferences, Discussion Paper, 1998-40 Federal Reserve Board.

Gabaix, Xavier, 2012, Variable rare disasters: An exactly solved framework for ten puzzles in macro-finance, Quarterly Journal of Economics 127, 645-700.

Guvenen, Fatih, 2009, A parsimonious macroeconomic model for asset pricing, Econometrica $77,1711-1750$.

Hansen, Lars Peter, John C. Heaton, and Nan Li, 2008, Consumption strikes back? Measuring long-run risk, Journal of Political Economy 116, 260-302.

Kaltenbrunner, Georg, and Lars A. Lochstoer, 2010, Long-run risk through consumption smoothing, Review of Financial Studies 23, 3190-3224.

Kreps, David M., and Evan L. Porteus, 1978, Temporal resolution of uncertainty and dynamic choice theory, Econometrica 46, 185-200.

Lazrak, Ali, 2004, Generalized stochastic differential utility and preference for information, Annals of Applied Probability 14, 2149-2175.

Marinacci, Massimo, and Luigi Montrucchio, 2010, Unique solutions for stochastic recursive utilities, Journal of Economic Theory 145, 1776-1804.

Mehra, Rajnish, and Edward C. Prescott, 1985, The equity premium: A puzzle, Journal of Monetary Economics 15, 145-161.

Obstfeld, Maurice, 1994, Evaluating risky consumption paths: The role of intertemporal substitutability, European Economic Review 38, 1471-1486.

Schroder, Mark, and Costis Skiadas, 1999, Optimal consumption and portfolio selection with stochastic differential utility, Journal of Economic Theory 89, 68-126.

Skiadas, Costis, 2008, Dynamic portfolio theory and risk aversion, in John R. Birge, and Vadim Linetsky, ed.: Handbooks in Operations Research and Management Science: Financial Engineering . vol. 15 (Elsevier North-Holland).

_ 2013, Smooth ambiguity aversion toward small risks and continuous-time recursive utility, Journal of Political Economy, forthcoming. 
Svensson, Lars E.O., 1989, Portfolio choice with non-expected utility in continuous time, Economics Letters 30, 313-317.

Tallarini, Thomas D. Jr., 2000, Risk-sensitive real business cycles, Journal of Monetary Economics 45, 507-532.

Uhlig, Harald, 2007, Leisure, growth and long run risk, Working Paper, University of Chicago.

Wachter, Jessica A., 2011, Can time-varying risk of rare disasters explain aggregate stock market volatility?, Working Paper, University of Pennsylvania.

Weil, Philippe, 1989, The equity premium puzzle and the risk-free rate puzzle, Journal of Monetary Economics 24, 401-421.

, 1990, Nonexpected utility in macroeconomics, Quarterly Journal of Economics 105, 29-42.

Zhang, Jianfeng, 2004, A numerical scheme for BSDEs, Annals of Applied Probability 14, 459-488. 


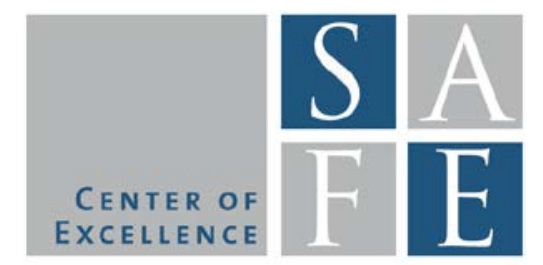

WORKING PAPER SERIES

\section{Recent Issues}

No. 16 Marius Ascheberg, Nicole Branger, Holger Kraft

No. 15 Holger Kraft, Claus Munk, Frank Thomas Seifried, Sebastian Wagner

No. 14 Dirk Bursian, Ester Faia

No. 13 Laurent E. Calvet, Paolo Sodini

No. 12 Marcel Bluhm, Ester Faia, J an Pieter Krahnen

No. 11 Nicole Branger, Holger Kraft, Christoph Meinerding

No. 10 Tim Eisert, Christian Eufinger

No. 9 Christian Eufinger, Andrej Gill

No. 8 Ignazio Angeloni, Ester Faia, Marco Lo Duca

No. 7 Matthieu Darraq Paries - Ester Faia - Diego Rodriguez Palenzuela

No. 6 Holger Kraft - Eduardo Schwartz - Farina Weiss

No. 5 Grigory Vilkov - Yan Xiao
When do J umps Matter for Portfolio Optimization?

Habits and Humps

Trust in the Monetary Authority

Twin Picks: Disentangling the Determinants of Risk-Taking in Household Portfolios

Endogenous Banks' Networks, Cascades and Systemic Risk

How Does Contagion Affect General Equilibrium Asset Prices?

Interbank network and bank bailouts: Insurance mechanism for non-insured creditors?

Basel III and CEO compensation in banks: Pay structures as a regulatory signal

Monetary Policy and Risk Taking

Bank and Sovereign Debt Risk Connection

Growth Options and Firm Valuation

Option-Implied Information and Predictability of Extreme Returns 